\title{
The Pristine Inner Galaxy Survey (PIGS) III: carbon-enhanced metal-poor stars in the bulge ${ }^{\star}$
}

\author{
Anke Arentsen, ${ }^{1} \dagger$ Else Starkenburg, ${ }^{2}$ David S. Aguado, ${ }^{3}$ Nicolas F. Martin,,${ }^{1,4}$ Vinicius M. Placco, ${ }^{5}$ \\ Raymond Carlberg, ${ }^{6}$, Jonay I. González Hernández, ${ }^{7,8}$ Vanessa Hill, ${ }^{9}$ Pascale Jablonka, ${ }^{10,11}$ \\ Georges Kordopatis, ${ }^{9}$ Carmela Lardo, ${ }^{12}$ Lyudmila I. Mashonkina, ${ }^{13}$ Julio F. Navarro, ${ }^{14}$ Kim A. Venn, ${ }^{14}$ \\ Sven Buder ${ }^{15,16}$, Geraint F. Lewis ${ }^{17}$, Zhen Wan ${ }^{17}$, Daniel B. Zucker ${ }^{18,19}$ \\ ${ }^{1}$ Université de Strasbourg, CNRS, Observatoire astronomique de Strasbourg, UMR 7550, F-67000 Strasbourg, France \\ ${ }^{2}$ Kapteyn Astronomical Institute, University of Groningen, Postbus 800, 9700 AV, Groningen, the Netherlands \\ ${ }^{3}$ Institute of Astronomy, University of Cambridge, Madingley Road, Cambridge CB3 OHA, UK \\ ${ }^{4}$ Max-Planck-Institut für Astronomie, Königstuhl 17, D-69117 Heidelberg, Germany \\ ${ }^{5}$ Community Science and Data Center/NSF's NOIRLab, 950 N. Cherry Ave., Tucson, AZ 85719, USA \\ ${ }^{6}$ Department of Astronomy \& Astrophysics, University of Toronto, Toronto, ON M5S 3H4, Canada \\ ${ }^{7}$ Instituto de Astrofísica de Canarias, Vía Láctea, 38205 La Laguna, Tenerife, Spain \\ ${ }^{8}$ Universidad de La Laguna, Departamento de Astrofísica, 38206 La Laguna, Tenerife, Spain \\ ${ }^{9}$ Université Côte d'Azur, Observatoire de la Côte d'Azur, CNRS, Laboratoire Lagrange, Nice, France \\ ${ }^{10}$ Institute of Physics, Laboratoire d'astrophysique, École Polytechnique Fédérale de Lausanne (EPFL), Observatoire, CH-1290 Versoix, Switzerland \\ ${ }^{11}$ GEPI, Observatoire de Paris, Université PSL, CNRS, Place Jules Janssen, F-92190 Meudon, France \\ ${ }^{12}$ Dipartimento di Fisica e Astronomia, Università degli Studi di Bologna, Via Gobetti 93/2, I-40129 Bologna, Italy \\ ${ }^{13}$ Institute of Astronomy of the Russian Academy of Sciences, Pyatnitskaya st. 48, 119017, Moscow, Russia \\ ${ }^{14}$ Department of Physics \& Astronomy, University of Victoria, Victoria, BC, V8W 3P2, Canada \\ ${ }^{15}$ Research School of Astronomy \& Astrophysics, Australian National University, ACT 2611, Australia \\ ${ }^{16}$ Center of Excellence for Astrophysics in Three Dimensions (ASTRO-3D), Australia \\ ${ }^{17}$ Sydney Institute for Astronomy, School of Physics, A28, The University of Sydney, NSW 2006, Australia \\ ${ }^{18}$ Department of Physics and Astronomy, Macquarie University, Sydney, NSW 2109, Australia \\ ${ }^{19}$ Macquarie University Research Centre for Astronomy, Astrophysics \& Astrophotonics, Sydney, NSW 2109, Australia
}

Accepted 2021 May 7. Received 2021 May 6; in original form 2021 April 14

\begin{abstract}
The most metal-deficient stars hold important clues about the early build-up and chemical evolution of the Milky Way, and carbon-enhanced metal-poor (CEMP) stars are of special interest. However, little is known about CEMP stars in the Galactic bulge. In this paper, we use the large spectroscopic sample of metal-poor stars from the Pristine Inner Galaxy Survey (PIGS) to identify CEMP stars $([\mathrm{C} / \mathrm{Fe}] \geqslant+0.7)$ in the bulge region and to derive a CEMP fraction. We identify 96 new CEMP stars in the inner Galaxy, of which 62 are very metal-poor $([\mathrm{Fe} / \mathrm{H}]<-2.0)$; this is more than a ten-fold increase compared to the seven previously known bulge CEMP stars. The cumulative fraction of CEMP stars in PIGS is $42_{-13}^{+14} \%$ for stars with [Fe/H] $<-3.0$, and decreases to $16_{-3}^{+3} \%$ for $[\mathrm{Fe} / \mathrm{H}]<-2.5$ and $5.7_{-0.5}^{+0.6} \%$ for $[\mathrm{Fe} / \mathrm{H}]<-2.0$. The PIGS inner Galaxy CEMP fraction for $[\mathrm{Fe} / \mathrm{H}]<-3.0$ is consistent with the halo fraction found in the literature, but at higher metallicities the PIGS fraction is substantially lower. While this can partly be attributed to a photometric selection bias, such bias is unlikely to fully explain the low CEMP fraction at higher metallicities. Considering the typical carbon excesses and metallicity ranges for halo CEMP-s and CEMP-no stars, our results point to a possible deficiency of both CEMP-s and CEMP-no stars (especially the more metal-rich) in the inner Galaxy. The former is potentially related to a difference in the binary fraction, whereas the latter may be the result of a fast chemical enrichment in the early building blocks of the inner Galaxy.
\end{abstract}

Key words: stars: chemically peculiar - stars: carbon - stars: Population II - Galaxy: bulge - Galaxy: formation - techniques: spectroscopic

\footnotetext{
* based on observations made with the Canada-France-Hawaii Telescope

(CFHT) and the Anglo-Australian Telescope (AAT)

$\dagger$ E-mail: anke.arentsen@ astro.unistra.fr
} 


\section{INTRODUCTION}

The Milky Way contains stars with a wide range of metallicities, covering different formation time scales and environments. The most metal-poor stars are typically expected to be among the oldest, and are mainly found in low-density environments such as the Galactic halo and dwarf galaxies. Metal-poor stars contain important clues about the First Stars and the earliest formation history of the Milky Way, which is why they have been searched for and studied extensively (see, e.g., Frebel \& Norris 2015, for a recent review).

A region in the Milky Way largely missing from the study of metalpoor stars has been the Galactic bulge. The halo is expected to have its highest density in the inner Galaxy, but it is an extremely challenging area to study. Metal-poor stars are highly outnumbered by metal-rich stars, and dust extinction obstructs and disturbs our view. However, thanks to efficient pre-selection methods and/or large surveys, the number of very metal-poor inner Galaxy stars identified and studied has been increasing in recent years (Ness et al. 2013; García Pérez et al. 2013; Howes et al. 2014, 2015, 2016; Casey \& Schlaufman 2015; Koch et al. 2016; Lamb et al. 2017; Lucey et al. 2019, 2021; Reggiani et al. 2020; Arentsen et al. 2020b).

One striking property of the most iron-poor stars in the halo is that they often show very large over-abundances of carbon, with the fraction of such carbon-enhanced metal-poor (CEMP) stars increasing with decreasing metallicity (e.g. Beers \& Christlieb 2005; Yong et al. 2013). In the Galactic halo, these CEMP stars are found to comprise $30 \%$ of stars with $[\mathrm{Fe} / \mathrm{H}]^{1}<-2$ and up to $80 \%$ of stars with $[\mathrm{Fe} / \mathrm{H}]<-4$ (Placco et al. 2014; Yoon et al. 2018), assuming a CEMP definition of $[\mathrm{C} / \mathrm{Fe}] \geqslant+0.7$.

Many of the CEMP stars with higher metallicities $([\mathrm{Fe} / \mathrm{H}]>$ -3.0) show enhancements in s-process elements and a very high absolute carbon abundance, fitting with the hypothesis that they are chemically enriched by mass transfer from a companion star that has gone through the asymptotic giant branch (AGB) phase (e.g., Lucatello et al. 2005; Bisterzo et al. 2010; Abate et al. 2015a). Because of their high s-process signature, these stars are referred to as CEMP-s stars (Beers \& Christlieb 2005). Indeed, a very high fraction of these stars have radial velocity variations consistent with them being in binary systems (Lucatello et al. 2005; Hansen et al. 2016b).

Most CEMP stars, however, show carbon enhancement without the accompanying s-process signature (for an overview see e.g. Norris et al. 2013). They are dominant among the most iron-poor stars, have lower absolute carbon values (Spite et al. 2013; Yoon et al. 2016) and they are typically not in binary systems (Starkenburg et al. 2014; Hansen et al. 2016a, although there are some interesting exceptions described in those works and in Arentsen et al. 2019). These CEMP-no stars were likely born with their carbon over-abundance, out of the early interstellar medium enriched by the First Stars. Two possible hypotheses for why the First Stars produce so much carbon are that they might have higher spin rates (Chiappini et al. 2006; Meynet et al. 2006) and/or that they might explode as mixing and fallback (faint) supernovae rather than ordinary supernovae (Umeda \& Nomoto 2003; Nomoto et al. 2013; Tominaga et al. 2014).

The fraction of CEMP stars and the relative number of CEMP$\mathrm{s}$ versus CEMP-no stars appear to vary throughout the Milky Way and its satellite galaxies. Relatively small samples of several hundred very metal-poor stars studied in detail have indicated trends of increasing CEMP fraction with increasing height from the Galactic

${ }^{1}[\mathrm{X} / \mathrm{Y}]=\log \left(N_{\mathrm{X}} / N_{\mathrm{Y}}\right)_{*}-\log \left(N_{\mathrm{X}} / N_{\mathrm{Y}}\right)_{\odot}$, where the asterisk subscript refers to the considered star, and $\mathrm{N}$ is the number density. In this work, $[\mathrm{Fe} / \mathrm{H}]$ and metallicity are used interchangably. plane (Frebel et al. 2006), and a larger relative number of CEMP-no stars further away into the halo (Carollo et al. 2014). Similar results are seen in recent work using tens of thousands of stars with low resolution spectroscopy from the Sloan Digital Sky Survey (SDSS) and the AAOmega Evolution of Galactic Structure (AEGIS) survey, which produced "carbonicity" maps and found the average [C/Fe] to increase with increasing distance from the Sun (Lee et al. 2017, 2019; Yoon et al. 2018). They also found that the relative number of CEMP-s stars is larger closer to the Sun, and that CEMP-no stars appear to become more dominant at larger distances. In the Milky Way satellites, an apparent lack of CEMP-no stars has been observed in dwarf spheroidal galaxies, whereas the CEMP-no stars are found in "usual" frequencies in ultra faint dwarf galaxies (e.g. Norris et al. 2010; Lai et al. 2011; Frebel et al. 2014; Skúladóttir et al. 2015). Because of these observations, some of these studies have suggested that the fraction of CEMP-no stars may be lower in more massive building blocks. However, others have suggested that this is simply a selection effect related to the varying metallicity distribution functions in the different types of dwarf galaxies (Salvadori et al. 2015). The carbonicity gradient in the halo can thus be related to the halo metallicity gradient and/or the type of building block that contributed to each part. To summarise, spatial metallicity and carbon trends can give us valuable insight into the early chemical and dynamical evolution of the Milky Way and its building blocks.

The Extremely Metal-poor BuLge stars with AAOmega (EMBLA) survey (Howes et al. 2014, 2015, 2016) was the first large metalpoor bulge survey, using metallicity-sensitive SkyMapper photometry (Bessell et al. 2011; Wolf et al. 2018) to select the most metalpoor candidates for spectroscopic follow-up. They derived the carbon abundances for 33 metal-poor stars (almost all $[\mathrm{Fe} / \mathrm{H}]<-2.0)$, and found only one of them to have a predicted natal $[\mathrm{C} / \mathrm{Fe}]$ (corrected for stellar evolution effects) above $[\mathrm{C} / \mathrm{Fe}]=+1.0$. This is in striking contrast with the much higher fraction of CEMP stars in the Galactic halo. A concern with that result is that the photometric SkyMapper selection could be biased against carbon-rich stars (shown in e.g. Da Costa et al. 2019; Chiti et al. 2020), since the wavelength region covered by their metallicity-sensitive narrow-band $v$ filter also contains a $\mathrm{CN}$ band. Given its major implications for early chemical evolution in our Galaxy, it is of importance that the inner Galaxy CEMP fraction is further studied with larger sample sizes and independent methods. Other previous metal-poor bulge studies either did not measure carbon and/or had very small sample sizes.

In this work, we present a complementary view of carbon abundances in the inner Galaxy from the Pristine Inner Galaxy Survey (PIGS, Arentsen et al. 2020b, hereafter A20b). Similar to EMBLA, PIGS also makes use of metallicity-sensitive photometry to select candidate stars, but a difference is that the employed narrow-band filter is significantly narrower than the SkyMapper $v$ filter - it is therefore expected to be less affected by surrounding $\mathrm{CN}$ and $\mathrm{CH}$ molecular bands. We make use of the low/intermediate-resolution spectroscopic follow-up of thousands of PIGS stars with measured metallicities and carbon abundances to study CEMP stars in the inner Galaxy. The relevant details about PIGS are presented in Section 2. We then describe the behaviour of carbon on the red giant branch in the PIGS sample and derive evolutionary carbon corrections for the PIGS $[\mathrm{C} / \mathrm{Fe}]$ determinations in Section 3. In Section 4 we present the discovery of many new CEMP stars in the inner Galaxy, and study the CEMP fraction as function of $[\mathrm{Fe} / \mathrm{H}]$. We discuss the interpretations and limitations of our results in Section 5, and summarise our conclusions in Section 6. 


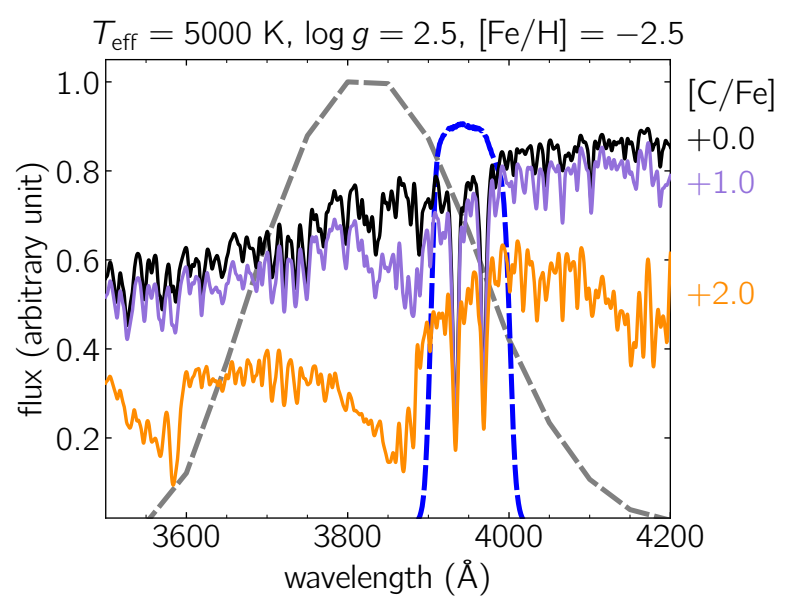

Figure 1. Filter curves for the SkyMapper $v$ (grey) and Pristine CaHK (blue) filters, plotted over synthetic spectra for three stars with the same stellar parameters (listed above the figure), but different $[\mathrm{C} / \mathrm{Fe}]$. The $\mathrm{CNO}$ abundances are fixed to "typical" values for CEMP-no stars (see the text for details). The Pristine filter is much narrower than the SkyMapper filter, and is expected to be less influenced by enhanced carbon.

\section{THE Pristine INNER GALAXY SURVEY (PIGS)}

\subsection{Survey overview}

For details on the Pristine Inner Galaxy Survey we refer the reader to A20b, the second paper in the PIGS series describing the survey photometry, selection for follow-up and spectroscopic analysis. Here, we only briefly summarise the relevant details. PIGS is an extension of the main Pristine survey, a photometric survey using the metallicity-sensitive narrow-band $\mathrm{CaHK}$ MegaCam filter on the Canada-France-Hawaii-Telescope (CFHT) to find and study the most metal-poor stars (for an overview, see Starkenburg et al. 2017). The $\mathrm{CaHK}$ filter centred on the strong $\mathrm{Ca} \mathrm{H} \& \mathrm{~K}$ stellar absorption lines is shown in Figure 1 together with the broader SkyMapper $v$-filter, on top of synthetic spectra of varying carbon abundance. The synthetic spectra were created using publicly available stellar atmosphere models from MARCS (Gustafsson et al. 2008; Plez 2008), combined with the Turbospectrum spectral synthesis code (Alvarez \& Plez 1998). We assumed $[\mathrm{C} / \mathrm{N}]=0$, and $[\mathrm{O} / \mathrm{Fe}]$ increasing with increasing $[\mathrm{C} / \mathrm{Fe}]$ (a typical signature for CEMP-no stars): $[\mathrm{O} / \mathrm{Fe}]=+0.6$ for carbon-normal stars and +1.3 and +1.9 for $[\mathrm{C} / \mathrm{Fe}]=+1.0$ and +2.0 , respectively.

The Pristine survey has an extensive spectroscopic follow-up campaign of its most promising candidates (Youakim et al. 2017; Caffau et al. 2017; Starkenburg et al. 2018; Aguado et al. 2019; Bonifacio et al. 2019; Venn et al. 2020; Caffau et al. 2020; Kielty et al. 2020), and it has a dedicated dwarf galaxy program (Longeard et al. 2018, 2020a,b). Instead of focusing on the Galactic halo and the satellites that reside in it, PIGS is looking towards the inner regions of the Galaxy - a far more challenging environment for a number of reasons. First, the bulge is on average much more metalrich, having a metallicity distribution that peaks only slightly below solar (around $\sim-0.2 /-0.5$ depending on the latitude, e.g. Ness et al. 2013), whereas in the halo the mean metallicity lies around $[\mathrm{Fe} / \mathrm{H}]=-1.6$ (e.g., Youakim et al. 2020). Secondly, the amount of dust and therefore of extinction is far greater towards the inner Galaxy than towards the Galactic halo, and it can be very inhomogeneous. The limited resolutions of available extinction maps and variations of the extinction law across the sky (Schlafly et al. 2016)

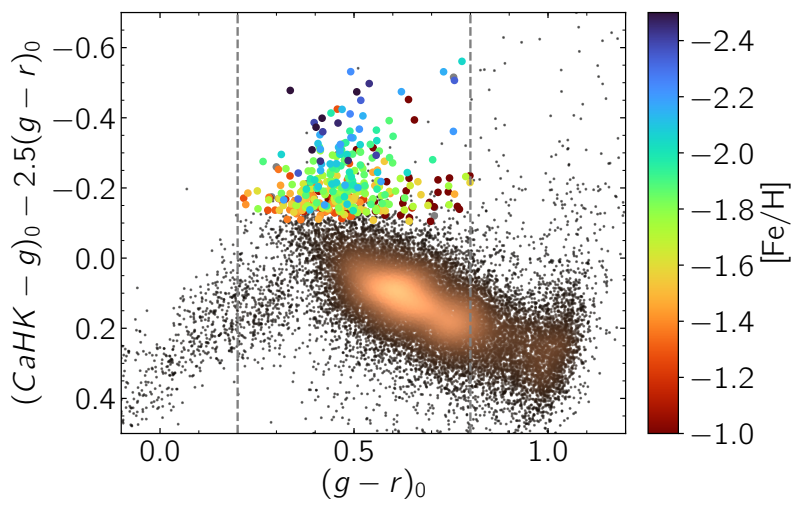

Figure 2. PIGS colour-colour diagram for a follow-up field selected with PS1. The field is located at $(l, b)=\left(7.6^{\circ}, 8.0^{\circ}\right)$, has a radius of $1^{\circ}$ and has an average $\mathrm{E}(\mathrm{B}-\mathrm{V})=0.51$. The background shows the density of all $\sim 22000$ stars passing our quality selection criteria in this field; the $\sim 350$ metal-poor candidates followed-up with AAT have been colour-coded by their spectroscopic $[\mathrm{Fe} / \mathrm{H}]$. The vertical lines indicate the colour cuts made for the follow-up selection.

severely hamper an accurate extinction correction. The bulge footprint of PIGS is chosen such that it avoids the most extincted regions; it surveys regions with $\mathrm{E}(\mathrm{B}-\mathrm{V}) \lesssim 0.7$ for the part of the sky bound by declination $>-30^{\circ}$ (because CFHT is a Northern Hemisphere facility) and $l$ and $b$ roughly between $-12^{\circ}$ and $+12^{\circ}$.

Much of the PIGS footprint has been followed-up with spectroscopic observations using AAOmega $+2 \mathrm{dF}$ at the AAT (Saunders et al. 2004; Lewis et al. 2002; Sharp et al. 2006). As discussed in A20b, the exact details of the target selection have varied throughout the progression of the survey. In all instances, however, the targets are selected from a colour-colour space including the $\mathrm{CaHK}$ and broad-band colours either from Gaia DR2 (Gaia Collaboration et al. 2018) or from PanSTARRS1 (PS1, Chambers et al. 2016). The colour-colour selection for an example field is shown in Figure 2. The broad-band photometry is necessary as a proxy for the temperature of the star, and a combination of broad-band colours and Pristine $\mathrm{CaHK}$ photometry is used as an indication of the metallicity. Because of unavoidable difficulties with differential reddening and the zeropoint of the $\mathrm{CaHK}$ photometry in this challenging Galactic region, we refrained from calculating photometric metallicities for each star. Instead, candidates were selected on a relative basis in each field and the fibres of the AAOmega spectrograph were filled from lower to higher expected metallicities, starting from the most metal-poor candidates located in the upper part of the colour-colour diagram. A20b already showed that this selection has an unprecedentedly high efficiency in selecting the most metal-poor populations in the inner Galaxy: $90 \%$ of the best candidates are confirmed to satisfy [Fe/H] $<-2.0$ from the spectroscopy, as do $60-75 \%$ of the next best selection box (depending on the magnitude of extinction in the field).

The first paper of the PIGS series (Arentsen et al. 2020a) used the spectroscopic follow-up observations to investigate the kinematical signature of metal-poor $([\mathrm{Fe} / \mathrm{H}]<-1.0)$ inner Galaxy stars and compared it with the stars at higher metallicities. It shows for the first time how the signature of Galactic rotation, observed in metal-rich stars, decreases with metallicity for metal-poor stars and completely disappears for stars with $[\mathrm{Fe} / \mathrm{H}]<-2.0$.

As we selected stars with a limited magnitude range of $13.5<$ 


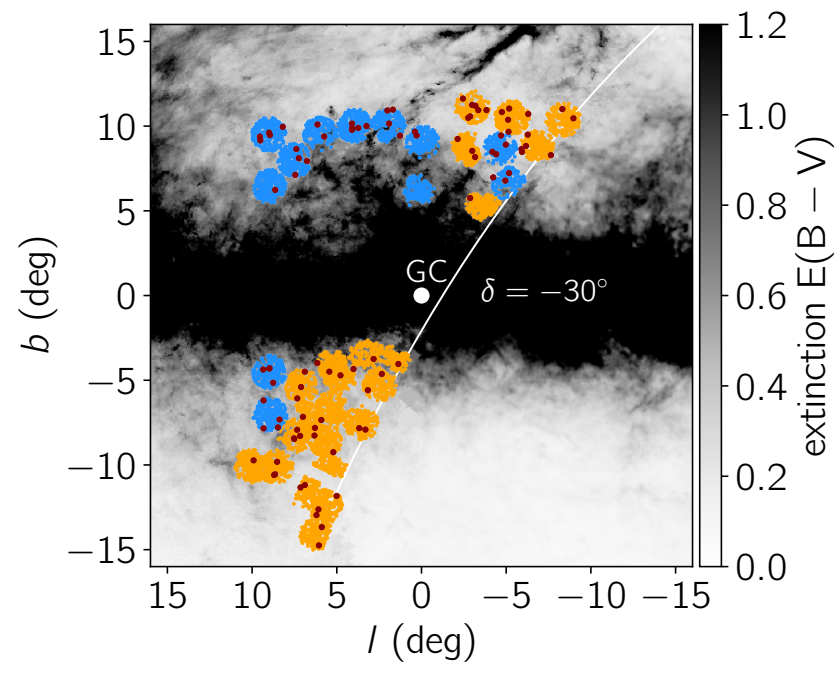

Figure 3. PIGS spectroscopic footprint for stars with $[\mathrm{Fe} / \mathrm{H}]<-1.5$, with orange fields already present in A20b and blue fields added in 2020. CEMP stars from this work are indicated as dark red points. The extinction comes from Green et al. (2019) above $\delta=-30^{\circ}$ and from Schlegel et al. (1998) below.

$G<16.5$ for Gaia, or $14.0<g<17.0$ for PS1, and we deselect foreground dwarf stars using Gaia parallax information (see A20b), the stars are expected to be roughly at the distance of the bulge. A kinematic analysis with Gaia data investigating the detailed orbits of the PIGS stars is planned for a future work.

The most recent PIGS follow-up coverage is shown in Figure 3, which includes 12 additional fields (observed in 2020) compared to A20b. Altogether, the PIGS AAOmega+2dF spectroscopic follow-up sample contains $\sim 12000$ stars with low/medium-resolution spectra covering $3700-5500 \AA$ (blue arm, R 1300) and 8400 - $8800 \AA$ (red arm, $\mathrm{R} \sim 11000$ ). The final sample (after quality cuts) contains $\sim 4800$ intermediate metal-poor (IMP) stars with $-2.0<[\mathrm{Fe} / \mathrm{H}]<-1.5$, and $\sim 1900$ very metal-poor (VMP) stars with $[\mathrm{Fe} / \mathrm{H}]<-2.0$. The median signal-to-noise ratio (SNR) per pixel is 19,57 and 50 for $4000-4100 \AA, 5000-5100 \AA$ and $8400-8800 \AA$, respectively.

\subsection{Stellar parameters and carbon abundances}

The PIGS spectra have been analysed with two independent pipelines $\left(\right.$ FERRE $^{2}$ and $\mathrm{ULySS}^{3}$ ), which yielded largely consistent results (A20b). In this work we will use the FERRE analysis, since it includes $[\mathrm{C} / \mathrm{Fe}]$ and extends to lower metallicities than the ULySS analysis. The method will be briefly summarised here.

The full-spectrum fitting code FERRE was employed for the derivation of the effective temperature $T_{\text {eff }}$, surface gravity $\log g$, metallicity $[\mathrm{Fe} / \mathrm{H}]$ and the carbon abundance $[\mathrm{C} / \mathrm{Fe}]$. We fit the observed spectra against a synthetic stellar model library, interpolating between the nodes with a cubic algorithm. The blue and red arm of the spectra are fit simultaneously to derive $T_{\text {eff }}, \log g,[\mathrm{Fe} / \mathrm{H}]$ and $[\mathrm{C} / \mathrm{Fe}]$. The reference synthetic grid is an extension of the carbonrich grid described in Aguado et al. (2017), based on Kurucz model

\footnotetext{
2 FERRE (Allende Prieto et al. 2006) is available from http://github. com/callendeprieto/ferre

${ }^{3}$ ULySS (Koleva et al. 2009) is available from http://ulyss. univ-lyon1.fr/
}

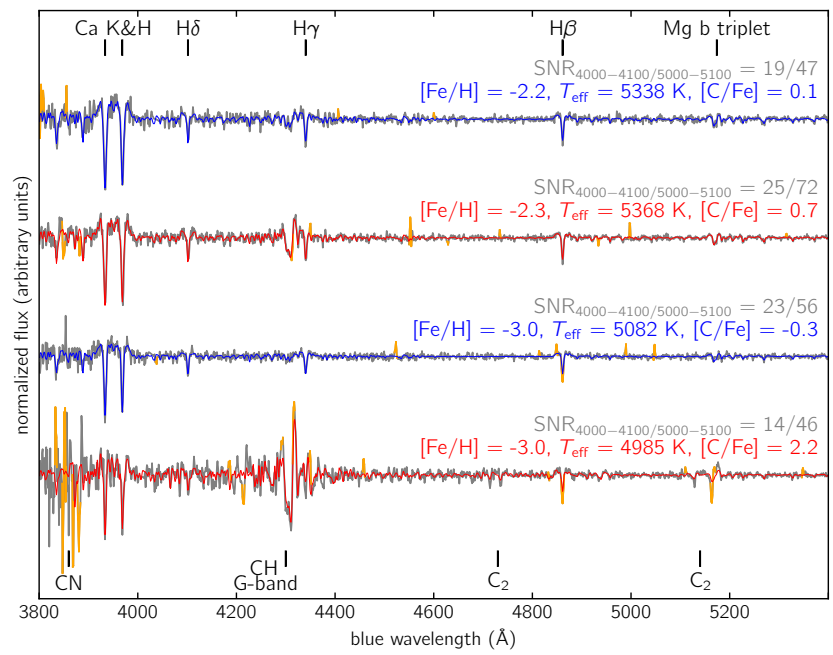

Figure 4. Example FERRE fits for two carbon-normal stars (blue) and two CEMP stars (red). The top two spectra have roughly the same metallicity and temperature, and the bottom two spectra as well (but a different metallicity and temperature as the top two). A running mean normalisation has been applied to both the observed and model spectra, and orange regions indicate outlier pixels excluded from the fit.

atmospheres (Mészáros et al. 2012) computed with the ASSET code (Koesterke et al. 2008). The new grid includes cooler stars (down to $T_{\text {eff }}=4500 \mathrm{~K}$ ) and extends to higher metallicities (up to $[\mathrm{Fe} / \mathrm{H}]=$ +0.5 ), with steps in $T_{\mathrm{eff}}, \log g,[\mathrm{Fe} / \mathrm{H}]$, and [C/Fe] of $250 \mathrm{~K}, 0.5 \mathrm{dex}$, $0.5 \mathrm{dex}$, and $1.0 \mathrm{dex}$, respectively. The alpha abundances are assumed to be enhanced following $[\alpha / \mathrm{Fe}]=+0.4$, which is typically appropriate for the metal-poor populations we focus on in this work. The spectra were self-consistently calculated using carbon and alphaenhanced atmospheres, adopting a microturbulence of $2 \mathrm{~km} \mathrm{~s}^{-1}$ (appropriate for giants). Four example fits are shown in Figure 4.

The comparison between the two analyses in A20b was used to derive final uncertainties on the stellar parameters. However, the ULySS analysis does not include $[\mathrm{C} / \mathrm{Fe}]$ as a parameter and, as such, we do not have a straightforward measurement of the external uncertainty to derive final uncertainties for $[\mathrm{C} / \mathrm{Fe}]$. We find that the internal uncertainties on $[\mathrm{C} / \mathrm{Fe}]$ are roughly two times larger than the internal uncertainties derived for $[\mathrm{Fe} / \mathrm{H}]$. Assuming that the external uncertainties on $[\mathrm{C} / \mathrm{Fe}]$ will follow the same trend, we quadratically add an uncertainty floor of $2 \times 0.11$ (the external $[\mathrm{Fe} / \mathrm{H}]$ uncertainty) $=0.22 \mathrm{dex}$ to all FERRE $[\mathrm{C} / \mathrm{Fe}]$ uncertainties. The median of the final $[\mathrm{C} / \mathrm{Fe}]$ uncertainties for our sample is $0.27 \mathrm{dex}$, and for $T_{\mathrm{eff}}$, $\log g$ and $[\mathrm{Fe} / \mathrm{H}]$ they are $148 \mathrm{~K}, 0.41$ dex and 0.14 dex, respectively.

\subsection{Degeneracy between carbon abundance and surface gravity}

Aguado et al. (2019) noted that there is a degeneracy between $\log g$ and $[\mathrm{C} / \mathrm{Fe}]$ in the parameter determination for VMP stars with FERRE. A bias in $\log g$ could therefore result in a bias in $[\mathrm{C} / \mathrm{Fe}]$. Their Figure 5 shows the difference in $[\mathrm{C} / \mathrm{Fe}]$ when they let $\log g$ be a free parameter versus when they set $\log g$ to a fixed value. There is an almost linear relation between the difference in $[\mathrm{C} / \mathrm{Fe}]$ and the $\log g$ for $1.0<\log g<3.5$, with an increase of $0.2 \mathrm{dex}$ in $[\mathrm{C} / \mathrm{Fe}]$ for a decrease in $\log g$ of 0.5 dex. It is challenging to derive $\log g$ from low/medium-resolution spectroscopy, especially for VMP stars since there are not many (gravity-sensitive) features in the spectrum. 

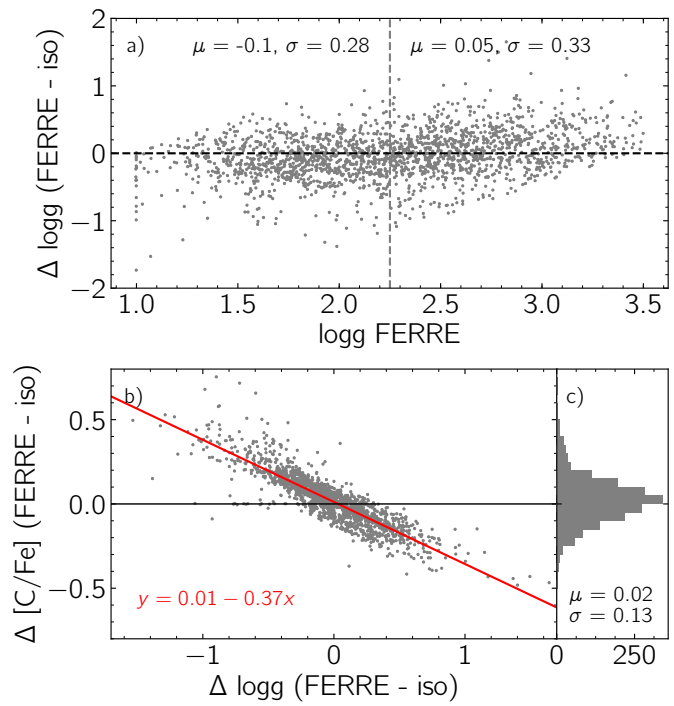

Figure 5. Tests of $\log g$ and $[\mathrm{C} / \mathrm{Fe}]$ for VMP stars $([\mathrm{Fe} / \mathrm{H}]<-2.0)$ in PIGS. Panel a) presents the difference between FERRE gravities and isochrone gravities, as function of the FERRE gravity. Panel b) shows the correlation between a difference in $\log g$ and the resulting difference in $[\mathrm{C} / \mathrm{Fe}]$. A linear fit to the points is presented in red. Panel c) shows the distribution of the $[\mathrm{C} / \mathrm{Fe}]$ differences, with the mean and standard deviation indicated.

The PIGS $\log g$ values are better determined than those from Aguado et al. (2019), because the spectra have higher SNR on average and because of the addition of the calcium triplet (this improves the results especially for lower gravity stars, see A20b). However, it is still possible that there could be some bias in $\log g$, and therefore in $[\mathrm{C} / \mathrm{Fe}]$.

To test for possible surface gravity/carbon abundance biases in the PIGS VMP sample, we compare the spectroscopic $\log g$ values to $\log g$ values from isochrones, and we perform a second FERRE analysis where we adopt the isochrone $\log g$. We used Yonsei-Yale isochrones (Demarque et al. 2004) of $12 \mathrm{Gyr}$ in a grid from $[\mathrm{Fe} / \mathrm{H}]=$ -2.0 to -3.5 with steps of $0.1 \mathrm{dex}$, and adopted the $\log g$ of the point on the isochrone red giant branch (RGB) closest to the FERRE $[\mathrm{Fe} / \mathrm{H}]$ and $T_{\text {eff. }}$ A minority of the PIGS stars are likely on the early AGB instead of the RGB, for which this procedure is not entirely valid. These stars will be discussed in more detail in Section 3. The difference between the $\log g$ values from FERRE and from the isochrones is shown in panel a) of Figure 5. They tend to agree well, with an average difference of -0.1 dex and 0.05 dex for the lower and upper RGB, respectively, and a standard deviation of $\sim 0.3 \mathrm{dex}$ around the average in both regimes.

We then rerun FERRE for the VMP stars with only $[\mathrm{C} / \mathrm{Fe}]$ as a free parameter, fixing $\log g$ to the isochrone value and fixing $T_{\text {eff }}$ and $[\mathrm{Fe} / \mathrm{H}]$ to their values from the initial FERRE analysis. These two parameters are less sensitive to $\log g$. Panel b) of Figure 5 shows the correlation between the difference in $\log g$ and the difference in the derived $[\mathrm{C} / \mathrm{Fe}]$, and panel c) presents the distribution of the difference in $[\mathrm{C} / \mathrm{Fe}]$. The correlation is similar as what was found by Aguado et al. (2019), but the average $\Delta[\mathrm{C} / \mathrm{Fe}]$ is only 0.02 dex (with a standard deviation of $0.13 \mathrm{dex}$ ). We therefore conclude that carbon abundances derived in this work are not biased due to biased surface gravities, and use only the purely spectroscopic parameters.

\section{STELLAR EVOLUTION ON THE GIANT BRANCH}

As stars evolve on the RGB, their surface carbon abundance changes. Since PIGS contains almost exclusively giant stars, we discuss this effect here. In the inner layers of an RGB star, carbon has been converted to nitrogen in the CNO cycle. While ascending the RGB, the star experiences mixing episodes, in which material from layers deeper in the star are transported to the stellar surface. The first dredge up occurs at the start of the RGB, which does not significantly change the surface $[\mathrm{C} / \mathrm{Fe}]$ for metal-poor stars (Vandenberg \& Smith 1988; Charbonnel 1994; Gratton et al. 2000). No additional mixing is expected to take place from standard theoretical models, but observations have shown that the surface abundance of carbon and nitrogen changes significantly on the RGB for metal-poor stars. The strength of this "extra mixing" appears to depend on the initial metallicity and carbon and nitrogen abundances, and it has been found to be stronger in metal-poor stars (e.g. Gratton et al. 2000; Stancliffe et al. 2009; Placco et al. 2014). One proposed mechanism is thermohaline mixing, which has been shown to reproduce the abundances in lowmass, low-metallicity evolved giants relatively well (Stancliffe et al. 2007; Charbonnel \& Lagarde 2010; Denissenkov 2010). Recently, Shetrone et al. (2019) used a large sample of stars from APOGEE to study metallicity-dependent mixing and extra mixing in field giant stars. They confirmed that the extra mixing is extremely sensitive to metallicity, and that current standard models cannot adequately explain this effect. In this section, we investigate the change of carbon abundance on the RGB in PIGS, and determine evolutionary $[\mathrm{C} / \mathrm{Fe}]$ corrections.

\section{1 $[\mathrm{C} / \mathrm{Fe}]$ in the Kiel diagram of PIGS}

It is possible to visualise the effect of extra mixing in PIGS on the Kiel diagram $\left(T_{\text {eff }}-\log g\right)$. To this end, we select stars with $\mathrm{SNR}_{4000-4100}>25$ to only include those with good quality carbon abundances. The median total uncertainty on $[\mathrm{C} / \mathrm{Fe}]\left(\epsilon_{[\mathrm{C} / \mathrm{Fe}]}\right)$ is 0.22 dex in this high SNR sample. The sample is split in two: the IMP stars with $-2.0<[\mathrm{Fe} / \mathrm{H}]<-1.5$ and the VMP stars with $[\mathrm{Fe} / \mathrm{H}]<-2.0$. We present Kiel diagrams colour-coded by $[\mathrm{C} / \mathrm{Fe}]$ for these two samples in Figure 6 . The average value of $[\mathrm{C} / \mathrm{Fe}]$ clearly decreases for stars higher up the RGB (with lower $\log g$ values), for both the IMP and the VMP stars. This is expected with what is known from the literature (e.g. Gratton et al. 2000; Placco et al. 2014), although it has not been observed directly in the bulge region before. A comparison of depletion of carbon along the RGB in PIGS with literature models is discussed Section 3.1.1.

There also appears to be a population of stars above the RGB that shows lower $[\mathrm{C} / \mathrm{Fe}]$ values (especially in the IMP sample). In the independent ULySS analysis of the spectra there are also two sequences in the $T_{\text {eff }}-\log g$ diagram. We hypothesise that these stars are in fact not RGB stars, but that they lie on the parallel early AGB instead. Early AGB stars are expected to have a lower carbon abundance than RGB stars because they have experienced extra mixing all the way up the RGB, before they became horizontal branch stars and subsequently AGB stars. We make a rough separation of both populations indicated by a solid line in Figure 6 (slightly different for the IMP and VMP stars). The relative number of AGB stars is discussed in Section 3.1.2.

\subsubsection{Carbon depletion with $\log g$}

Panel a) of Figure 7 presents the mean trends of [C/Fe] with $\log g$ for the IMP and VMP RGB stars. The trend of decreasing $[\mathrm{C} / \mathrm{Fe}]$ with 


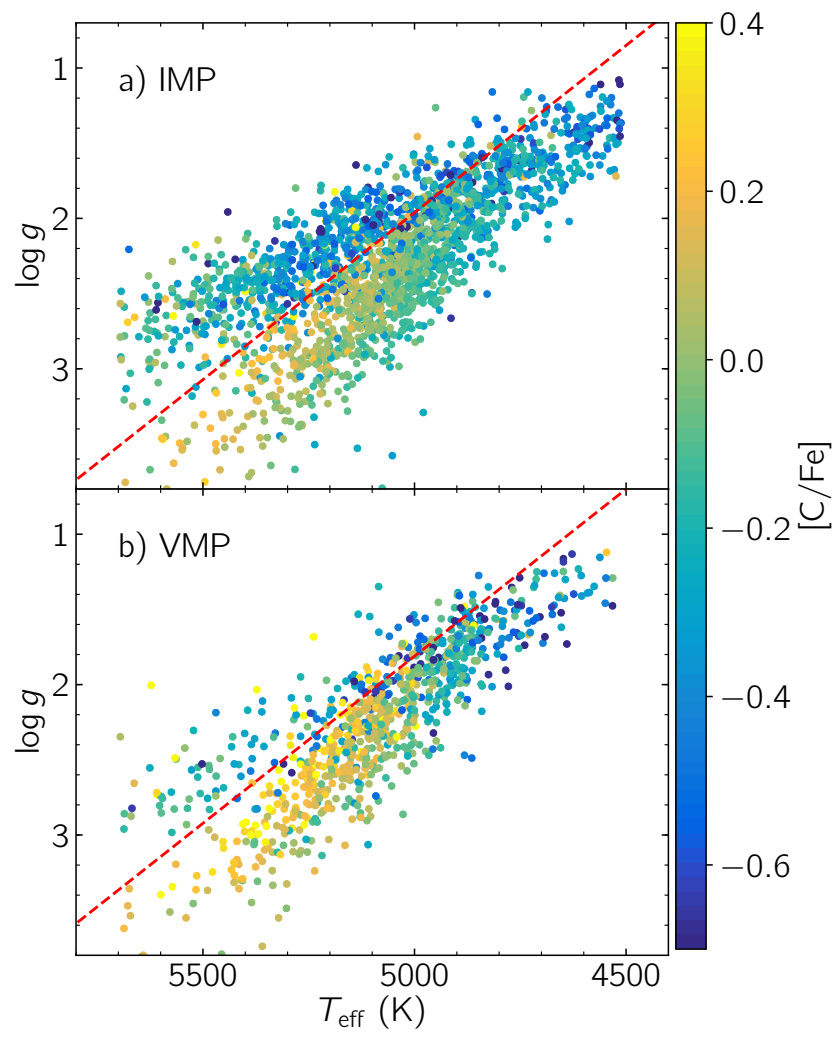

Figure 6. Kiel diagram of PIGS giants with $\mathrm{SNR}_{4000-4100}>25, T_{\text {eff }}<$ $5700 \mathrm{~K}$ and $[\mathrm{C} / \mathrm{Fe}]<+0.7$, for stars with $-2.0<[\mathrm{Fe} / \mathrm{H}]<-1.5$ (IMP, top panel) and $[\mathrm{Fe} / \mathrm{H}]<-2.0$ (VMP, bottom panel), colour-coded by $[\mathrm{C} / \mathrm{Fe}]$. The dotted red lines indicate a rough separation between the RGB and AGB stars.

the ascension on the RGB is clearly visible. Additionally, we find that the average $[\mathrm{C} / \mathrm{Fe}]$ for the unevolved VMP stars is $\sim 0.1$ dex higher than for the IMP stars. A trend of increasing $[\mathrm{C} / \mathrm{Fe}]$ with decreasing $[\mathrm{Fe} / \mathrm{H}]$ is also present in metal-poor halo samples (e.g., Roederer et al. 2014; Amarsi et al. 2019), although the latter authors show that the trend could largely be the result of 3D/non-local thermodynamic equilibrium (non-LTE) effects. Most literature studies, however, do not correct for $3 \mathrm{D}$ and/or non-LTE effects. We therefore assume that it is safe to compare our results with those of others, as long as the stars are in a similar range of the parameter space.

Also shown are predictions for the extra depletion of carbon in RGB stars, from the models by Placco et al. (2014) smoothed by the PIGS uncertainties. For the IMP stars we adopted $[\mathrm{Fe} / \mathrm{H}]=-1.75$ and natal $[\mathrm{C} / \mathrm{Fe}]=0.0$, and for the VMP stars $[\mathrm{Fe} / \mathrm{H}]=-2.3$ and natal $[\mathrm{C} / \mathrm{Fe}]=+0.1$. In the original Placco et al. (2014) models, the extra depletion of carbon starts around $\log g=2.0$ and increases steeply with decreasing $\log g$. However, the uncertainties of $[\mathrm{C} / \mathrm{Fe}]$ and especially $\log g$ in PIGS are significant, spreading out this sharp feature. We took one thousand draws around each of the original model points from normal distributions with standard deviations equal to the typical PIGS uncertainties in $\log g$ and $[\mathrm{C} / \mathrm{Fe}]$ to mimic this effect. For the high SNR sample used here, those uncertainties are $0.37 \mathrm{dex}$ and $0.22 \mathrm{dex}$ for $\log g$ and $[\mathrm{C} / \mathrm{Fe}]$, respectively. After this correction for the uncertainties, the models and the data agree well with each other. The carbon depletion appears to be slightly higher in the data than in the models for $2.5>\log g>1.7$, which could be

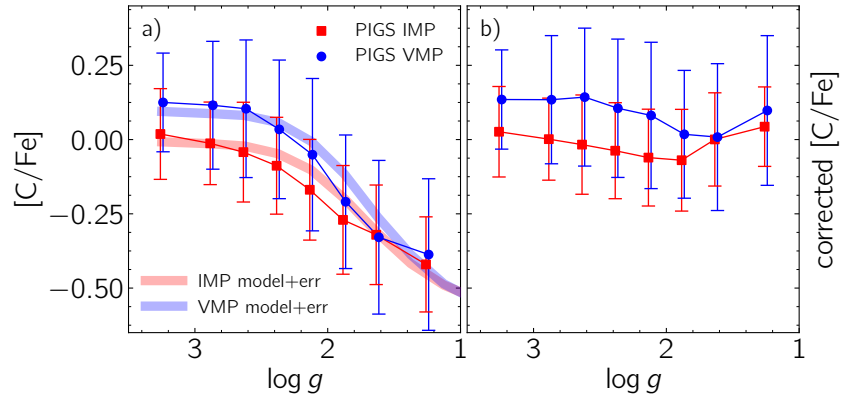

Figure 7. Left: mean $[\mathrm{C} / \mathrm{Fe}]$ versus $\log g$ in the IMP and VMP samples of RGB stars (stars below the dotted lines in Figure 6). The means are determined using a $5 \sigma$ clipping of outliers, the error bars represent the standard deviation in each $\log g$ bin. Also shown are theoretical predictions from the models by Placco et al. (2014), assuming typical parameters and uncertainties for stars in our IMP and VMP samples (see the main text for details). Right: mean $[\mathrm{C} / \mathrm{Fe}]$ corrected for evolutionary effects versus $\log g$.

the result of some (carbon-poor) AGB contamination on the RGB in this $\log g$ range (see also Figure 6).

We confirm that the evolutionary carbon depletion in our observations follows the theoretical predictions from Placco et al. (2014). The derivation of evolutionary carbon corrections for each individual star in the PIGS sample will be described in Section 3.2.

\subsubsection{Asymptotic giant branch (AGB) stars}

In the IMP sample, the total number of RGB stars is roughly the same as the number of AGB stars. Given that stars generally live longer on the RGB than on the AGB, this seems like a large fraction of AGB stars at face value. To gain a more quantitative understanding, we roughly estimate the expected occurrence ratio of such stars using two models: a MIST evolutionary track $^{4}$ (Dotter 2016; Choi et al. 2016) and a simulated stellar population from PARSEC ${ }^{5}$ (Bressan et al. 2012).

We limit the data and the models to the same $T_{\text {eff }}-\log g$ range $(4900-5200 \mathrm{~K}$ and $\log g<2.5$, where both the RGB and AGB samples are expected to be most complete) and select a narrow range in observed metallicities $( \pm 0.1 \mathrm{dex})$ around the model $[\mathrm{Fe} / \mathrm{H}]=$ -1.75 . In the data, $29 \%$ of the stars lie above the dotted RGB/AGB separation line in Figure 6. Within the selected parameter range in the models, stars spend $13 \%$ of their time on the AGB on a MIST evolutionary track of a $0.8 M_{\odot}$, and $13 \%$ of the stars in a $12 \mathrm{Gyr}$ old PARSEC simulated population of $10^{5} \mathrm{M}_{\odot}$ with a Kroupa (2001, 2002) IMF live on the AGB. Each of these numbers can change by a few percent depending on the chosen parameter limits.

There is a difference in relative number of AGB stars between the models (13\%) and the data (29\%), but for a simple estimate such as this it is not unacceptable. First, our separation of the data into RGB or AGB star is very rough, and the introduced cuts in $T_{\text {eff }}$ and $\log g$ for the comparison potentially bias the results. Furthermore, in reality, there is a range of ages and masses of stars in the inner Galaxy (and possibly helium abundances), complicating a model comparison. Additionally, evolutionary tracks and simulated stellar populations are not perfect. More detailed comparisons with for example observed globular clusters may be useful, but are beyond the scope of this work.

\footnotetext{
${ }^{4}$ http://waps.cfa.harvard.edu/MIST/interp_tracks.html

5 http://stev.oapd.inaf.it/cmd
} 
Another possibility is that our photometric target selection favours the carbon-poor AGB stars over carbon-normal stars, due to weaker carbon features in the spectra, although this effect is likely too small.

\subsection{Deriving evolutionary corrections}

The evolutionary carbon correction is stronger for stars with lower $\log g$ and stars of lower $[\mathrm{Fe} / \mathrm{H}]$, which is visible in panel a) of Figure 7. It is less strong for very carbon-rich stars (Placco et al. 2014). These three parameters, $\log g,[\mathrm{Fe} / \mathrm{H}]$ and $[\mathrm{C} / \mathrm{Fe}]$, are the input for the derivation of the corrections.

Including stellar parameter uncertainties in the derivation of the corrections is crucial, especially since the correction depends strongly on $\log g$ and the PIGS uncertainties in $\log g$ are nonnegligible. For each star, we take 100 draws from normal distributions around each of the parameters with widths equal to their total uncertainties, and compute carbon corrections for each of them. The mean of these 100 carbon corrections is adopted as the final carbon-correction for each star. We checked what happened when only varying one of the stellar parameters while keeping the other two constant, from which we concluded that the carbon corrections are affected the most by including uncertainties for $\log g$, and the differences for $[\mathrm{Fe} / \mathrm{H}]$ and $[\mathrm{C} / \mathrm{Fe}]$ are minor.

The average $[\mathrm{C} / \mathrm{Fe}]$ as function of $\log g$ for carbon-corrected RGB stars is shown in panel b) of Figure 7 . The trend is largely flat, indicating that the carbon-corrections are properly determined. There appears to be a small dip around $\log g=2.0$ (although it is consistent within the uncertainties), which potentially indicates contamination by AGB stars.

The carbon corrections are only valid for RGB stars, they underestimate the depletion of carbon for AGB stars. We experimented with adopting tip-of-the-RGB $\log g$ values for AGB stars (stars above the dashed line in Figure 6) to derive the correction with those. However, we found that this typically over-estimates the carbon-correction: the AGB stars end up with a higher average $[\mathrm{C} / \mathrm{Fe}]$ than the RGB stars. Additionally, it is difficult to decide for each individual star whether it is an AGB or an RGB star. Finally, this analysis depends on the correctness of the isochrones regarding the location of the tip of the RGB. At low $\log g$, the carbon-correction is very sensitive to the exact $\log g$ so small deviations have a large impact. It also depends on the assumption that no additional mixing happens between the tip of the RGB and early AGB, which is not known. Taking these considerations into account, we decide to not derive special corrections for the AGB stars but adopt the normal correction, considering it a lower limit. For VMP stars, the relative number of AGB stars is small.

\section{CEMP STARS IN PIGS}

The main goal of this work is to investigate the CEMP stars in PIGS. In this section, we report the discovery of a large number of CEMP stars in the inner Galaxy. We also discuss the CEMP fraction as a function of the metallicity, compared to the halo.

\subsection{New CEMP stars}

Throughout this work, the CEMP definition we adopt is $[\mathrm{C} / \mathrm{Fe}] \geqslant$ +0.7 . Among a sample of 8652 cool metal-poor giant stars in PIGS with $[\mathrm{Fe} / \mathrm{H}]<-1.0, T_{\text {eff }}<5700 \mathrm{~K}$ and $\log g<3.5$ we identify 96 CEMP stars, 47 of which have $[\mathrm{C} / \mathrm{Fe}] \geqslant+1.0$. For $[\mathrm{Fe} / \mathrm{H}]<$ -2.0 , these numbers are 62 and 36, respectively, out of 1836 stars. The $[\mathrm{C} / \mathrm{Fe}]$ was corrected for evolutionary effects as described in
Section 3. All of the PIGS CEMP stars are new discoveries, and they increase the total sample of known CEMP stars in the inner Galaxy more than ten-fold.

Previously, only two CEMP stars were reported in the bulge region: one CEMP-s star (Koch et al. 2016) and one CEMP-no star (in EMBLA, Howes et al. 2015, 2016). When applying Placco et al. (2014) evolutionary carbon corrections to the EMBLA sample and adopting the $[\mathrm{C} / \mathrm{Fe}] \geqslant+0.7$ CEMP definition, we find that three more stars can be classified as CEMP, all with $[\mathrm{Fe} / \mathrm{H}]<-3.0$ and low $[\mathrm{Ba} / \mathrm{Fe}]$ (hence these are CEMP-no stars). We consider two additional stars with $[\mathrm{Fe} / \mathrm{H}]=-1.97$ and -2.47 and $[\mathrm{C} / \mathrm{Fe}]=+0.69$ as CEMP stars. The more metal-rich of these also has a high $[\mathrm{Ba} / \mathrm{Fe}]$ and can therefore be classified as a CEMP-s star. This brings the total number of previously known inner Galaxy CEMP stars to seven.

The PIGS stars passing the CEMP criterion are shown as black circles on the $[\mathrm{Fe} / \mathrm{H}]-\mathrm{A}(\mathrm{C})^{6}$ diagram in Figure 8 . As detailed in the introduction of this paper, the different sub-populations of CEMP stars (CEMP-no and CEMP-s stars) show a typical separation in $\mathrm{A}(\mathrm{C})$ space. The previously known CEMP stars in the inner Galaxy (and a slightly more metal-rich $\mathrm{CH}$ star from Koch et al. 2016) are represented by coloured star symbols in Figure 8, together with a large sample of giant halo CEMP stars compiled by Yoon et al. (2016). Most of the CEMP stars in PIGS are found around $[\mathrm{Fe} / \mathrm{H}]=-2.0$, largely because that is where the metallicity distribution function of the PIGS follow-up peaks (as shown by the histogram in the bottom right corner).

The CEMP stars with $[\mathrm{Fe} / \mathrm{H}]<-2.0$ are spread relatively evenly across the giant branch, although they tend to have slightly lower $\log g$ compared to the carbon-normal stars. The CEMP stars with $-2.0<[\mathrm{Fe} / \mathrm{H}]<-1.5$, however, are mainly (23/32) located at relatively high temperatures and low gravities, in the early AGB regime. This could be the result of selection effects against carbonrich stars in the PIGS photometric selection which are stronger for cooler and more metal-rich stars, although it is not clear that such effects would be strong enough to create such a difference.

In the absence of s-process abundance information ${ }^{7}$ or any radial velocity monitoring for our stars, a different strategy is needed to classify the newly discovered CEMP stars. A general classification can be made based on the $\mathrm{A}(\mathrm{C})$ and $[\mathrm{Fe} / \mathrm{H}]$ of the stars (Spite et al. 2013; Bonifacio et al. 2015; Yoon et al. 2016). The latter authors suggest three different groups of CEMP stars: the Group I stars largely contain CEMP-s stars and are found at high $\mathrm{A}(\mathrm{C})(>7.1)$ and higher metallicity, and the Group II and III stars contain largely CEMP-no stars and are located at lower $\mathrm{A}(\mathrm{C})$ and/or lower metallicity. A rough separation of these groups has been indicated with dashed-dotted orange lines in Figure 8. The authors showed that the contamination of CEMP-no stars in Group I and CEMP-s stars in Groups II and III is small, although there are some "anomalous" stars which they discuss in detail. They also discuss possible different origins for the Group II and III CEMP-no stars. Others have used a higher A(C) value for the CEMP-s/no separation (Bonifacio et al. 2015; Lee et al. 2019), but those samples contain largely main-sequence turn-off stars whereas the Yoon et al. (2016) sample has a larger number of giants. Since PIGS contains solely giants, we adopt the Yoon et al. (2016) separation. We find that 72 stars lie in the CEMP-s region, and 24 in the CEMP-no part of the diagram. It is noteworthy that most of the

$6 A(\mathrm{C})=\log \epsilon(C)=\log \left(N_{C} / N_{H}\right)+12$
7 There are two strong lines of $\mathrm{Sr}$ and one of Ba in the blue arm spectra, but
due to the combination of low resolution, sometimes low $\mathrm{S} / \mathrm{N}$ and very low
metallicity, we cannot measure them for any of the stars with $[\mathrm{Fe} / \mathrm{H}]<-2.0$. 


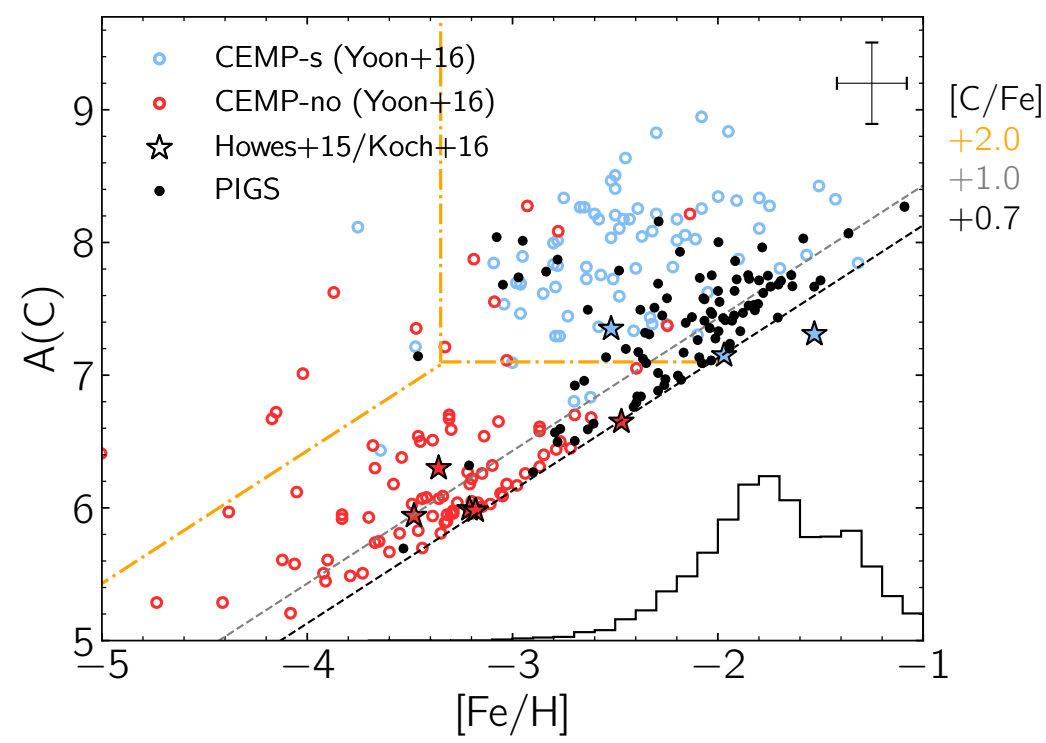

Figure 8. $\mathrm{A}(\mathrm{C})-[\mathrm{Fe} / \mathrm{H}]$ diagram for CEMP stars, with the new PIGS stars in black, a literature halo sample from Yoon et al. (2016) as red and blue circles (including only giant stars with $T_{\text {eff }}<5500 \mathrm{~K}$ and $\log g<3.5$ ), and the seven previously known bulge CEMP stars and one CH star from Howes et al. (2015) and Koch et al. (2016) with star symbols, coloured according to their CEMP class (see Section 5.2.2 for a discussion on the Howes et al. stars). Two dashed lines of constant $[\mathrm{C} / \mathrm{Fe}]$ are shown: $[\mathrm{C} / \mathrm{Fe}]=+0.7$ in black is our CEMP criterion and $[\mathrm{C} / \mathrm{Fe}]=+1.0$ (sometimes used in the literature) in grey. The dashed-dotted orange lines roughly separate the CEMP stars according to the Yoon et al. (2016) classification, with their Group I stars on the top right (mainly CEMP-s stars) and the Group III and II stars (mainly CEMP-no stars) on the top left and the bottom, respectively. These lines converge at $\mathrm{A}(\mathrm{C})=7.1,[\mathrm{C} / \mathrm{Fe}]=+2.0$ and $[\mathrm{Fe} / \mathrm{H}]=-3.35$. The metallicity distribution function of the 8652 PIGS stars with $[\mathrm{Fe} / \mathrm{H}]<-1.0$ in the same $T_{\text {eff }}$ and $\log g$ range as the CEMP stars is represented by the histogram. Typical uncertainties on the PIGS $[\mathrm{Fe} / \mathrm{H}]$ and $\mathrm{A}(\mathrm{C})$ are shown in the top-right corner.

CEMP-s candidates lie relatively close to the $[\mathrm{C} / \mathrm{Fe}]=+0.7$ line, whereas the literature sample does not and has much higher $[\mathrm{C} / \mathrm{Fe}]$ values. Most of the CEMP-no candidates in PIGS also lie close to the $[\mathrm{C} / \mathrm{Fe}]=+0.7$ line, but here it is more consistent with the distribution of halo CEMP-no stars. The star around $[\mathrm{Fe} / \mathrm{H}]=-3.5$ and $\mathrm{A}(\mathrm{C})=7.1$ is the only candidate Group III CEMP-no star.

\subsection{The CEMP fraction}

In the halo, the CEMP fraction is $~ 30 \%$ for stars with $[\mathrm{Fe} / \mathrm{H}]<-2.0$ and rises quickly for lower metallicity stars (e.g., Placco et al. 2014, and references therein). For a quantitative comparison between the distribution of CEMP stars in PIGS and the Galactic halo, we derive the cumulative CEMP fraction as a function of metallicity. We select VMP stars in PIGS with $4600 \mathrm{~K}<T_{\text {eff }}<5500 \mathrm{~K}$, a regime where the carbon abundances are expected to be the most reliable. Stars with $\epsilon_{[\mathrm{C} / \mathrm{Fe}]}>0.5 \mathrm{dex}$ are discarded. We draw 1000 samples from the distributions of $[\mathrm{Fe} / \mathrm{H}],[\mathrm{C} / \mathrm{Fe}]$ and their uncertainties, and adopt the median of the 1000 CEMP fractions and uncertainties as our final values $^{8}$.

The $[\mathrm{C} / \mathrm{Fe}]$ as a function of $[\mathrm{Fe} / \mathrm{H}]$ for the selected sample is presented in panel a) of Figure 9, with the PIGS cumulative percentage of CEMP stars below different $[\mathrm{Fe} / \mathrm{H}]$ values indicated in black. For $[\mathrm{Fe} / \mathrm{H}]<-3.0,-2.5,-2.0$, we find CEMP fractions of $42_{-13}^{+14} \%$, $16_{-3}^{+3} \%, 5.7_{-0.5}^{+0.6} \%$, respectively. Panel b) presents a comparison of the cumulative CEMP fraction in PIGS with the halo fraction from Placco et al. (2014) (including the halo fraction separated for CEMP$\mathrm{s}$ and CEMP-no stars). The PIGS fraction appears compatible with

8 The uncertainties on the fractions are computed using Wilson (1927) score confidence intervals of $1 \sigma$, as in e.g. Yoon et al. (2018).
Table 1. PIGS cumulative CEMP fraction for different $[\mathrm{C} / \mathrm{Fe}]$ limits

\begin{tabular}{lccc}
\hline$[\mathrm{Fe} / \mathrm{H}]$ & {$[\mathrm{C} / \mathrm{Fe}] \geqslant+0.5$} & {$[\mathrm{C} / \mathrm{Fe}] \geqslant+0.7$} & {$[\mathrm{C} / \mathrm{Fe}] \geqslant+1.0$} \\
\hline-3.25 & $50.0_{-22.4}^{+22.4} \%$ & $50.0_{-22.4}^{+22.4} \%$ & $25.0_{-15.0}^{+25.0} \%$ \\
-3.0 & $50.0_{-13.9}^{+13.9} \%$ & $41.7_{-13.0}^{+14.3} \%$ & $33.3_{-11.9}^{+14.4} \%$ \\
-2.75 & $40.0_{-6.7}^{+7.1} \%$ & $28.0_{-5.9}^{+6.7} \%$ & $20.0_{-5.0}^{+6.2} \%$ \\
-2.5 & $28.1_{-3.3}^{+3.6} \%$ & $16.4_{-2.6}^{+3.0} \%$ & $8.8_{-1.9}^{+2.4} \%$ \\
-2.25 & $19.1_{-1.5}^{+1.6 \%}$ & $9.5_{-1.1}^{+1.2} \%$ & $4.1_{-0.7}^{+0.9} \%$ \\
-2.0 & $13.3_{-0.8}^{+0.8} \%$ & $5.7_{-0.5}^{+0.6} \%$ & $2.1_{-0.3}^{+0.4} \%$ \\
\hline
\end{tabular}

the overall literature fraction for $[\mathrm{Fe} / \mathrm{H}]<-3.0$. For $[\mathrm{Fe} / \mathrm{H}]<-2.0$, however, the CEMP fraction in PIGS is strongly discrepant with the halo fraction which is about six times larger. Table 1 presents the cumulative CEMP fraction in PIGS for different carbon-enhanced definitions $([\mathrm{C} / \mathrm{Fe}] \geqslant+0.5,+0.7,+1.0)$, for reference.

The orange line in panel a) of Figure 9 can serve as a rough division between CEMP-s and CEMP-no stars. The CEMP stars with $-2.5<[\mathrm{Fe} / \mathrm{H}]<-2.0$ mostly lie in the CEMP-s regime, but at lower metallicities the sample appears approximately equally split between CEMP-s and CEMP-no. However, the sample sizes are small and higher resolution spectroscopic follow-up is necessary to properly assign the stars to either category - we therefore refrain from presenting separate CEMP-no and CEMP-s fractions here.

\subsection{Face-value distribution of PIGS CEMP stars}

PIGS has increased the number of known CEMP stars in the inner Galaxy by more than a factor of ten. However, the face-value dis- 

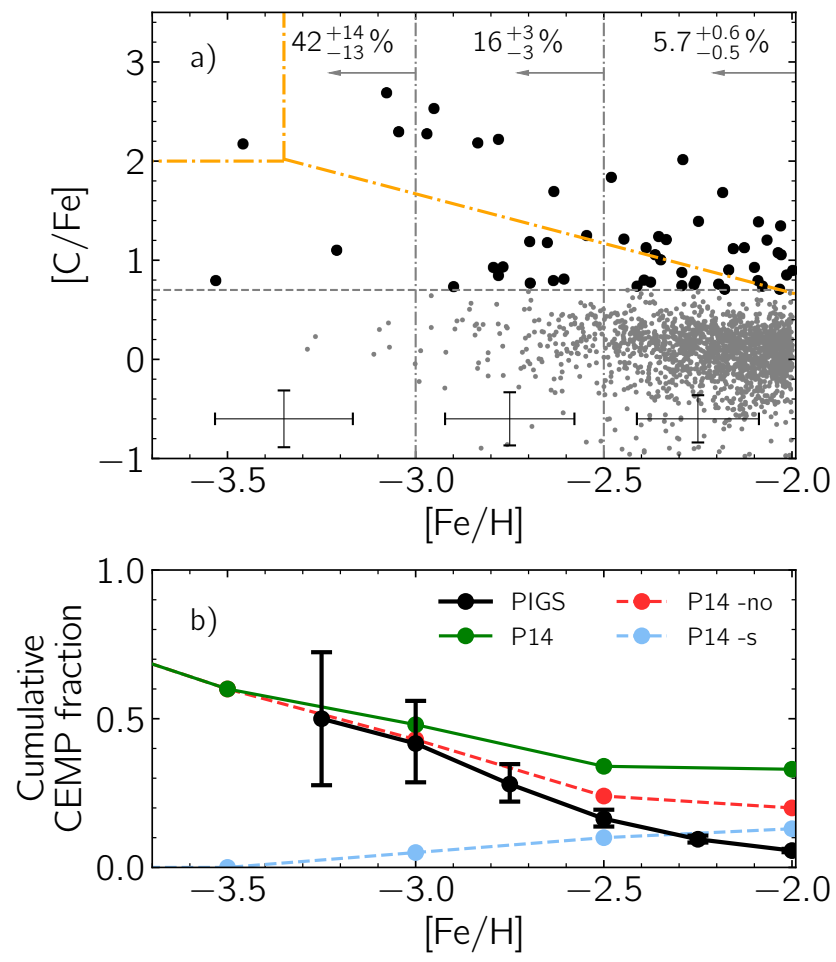

Figure 9. Top: $[\mathrm{Fe} / \mathrm{H}]$ versus [C/Fe] for PIGS stars with $4600 \mathrm{~K}<T_{\text {eff }}<$ $5500 \mathrm{~K}$. The horizontal dashed line indicates the CEMP criterion at $[\mathrm{C} / \mathrm{Fe}]=$ +0.7 , the CEMP stars (which are above this line) are shown with larger black symbols. The orange dashed lines are the same as in Figure 8. The PIGS cumulative CEMP fraction below different metallicities (vertical dot-dashed lines) is indicated in black. Typical uncertainties on $[\mathrm{Fe} / \mathrm{H}]$ and $[\mathrm{C} / \mathrm{Fe}]$ are shown on the bottom of each metallicity range. Bottom: PIGS cumulative CEMP fraction as function of $[\mathrm{Fe} / \mathrm{H}]$ for the same sample. The halo CEMP fraction from Placco et al. (2014) is shown in green, and separated into the fraction of CEMP-no (red dashed) and CEMP-s (blue dashed) stars.

tribution of candidate CEMP stars in PIGS on the $[\mathrm{Fe} / \mathrm{H}]$ vs. $\mathrm{A}(\mathrm{C})$ diagram is discrepant compared to the halo. There is a lack of very carbon-rich stars with $\mathrm{A}(\mathrm{C})>8.0$, especially for $[\mathrm{Fe} / \mathrm{H}]$ around -2.5 and higher - in the regime of the CEMP-s stars. The CEMP fraction in PIGS is lower than the fraction known from the Galactic halo, except for $[\mathrm{Fe} / \mathrm{H}]<-3$.0. If the PIGS selection is unbiased and the inner Galaxy CEMP fraction were the same as in the halo, we would have expected to find around $600 \mathrm{CEMP}$ stars with $[\mathrm{Fe} / \mathrm{H}]<-2.0$ in PIGS (33\% of 1900 stars), whereas we found only 62 . The difference is less striking for $[\mathrm{Fe} / \mathrm{H}]<-2.5$, where we would have expected to find $~ 60$ CEMP stars instead of the 20 in PIGS.

How many of the CEMP stars in PIGS could be "accidental" - simply the outliers of a Gaussian $[\mathrm{C} / \mathrm{Fe}]$ distribution? We fit a Gaussian to the $[\mathrm{C} / \mathrm{Fe}]$ distribution in Figure 9, excluding $3 \sigma$ outliers and stars above the RGB/AGB separation line in Figure 6. For the higher metallicity stars $(<-2.5<[\mathrm{Fe} / \mathrm{H}]<-2.0)$, the mean $[\mathrm{C} / \mathrm{Fe}]$ is 0.07 with a sigma of $0.20 \mathrm{dex}$. From such a distribution, one would expect only $0.1 \%$ of the stars to have $[\mathrm{C} / \mathrm{Fe}] \geqslant+0.7$. For the number of stars in PIGS in this metallicity range used in the CEMP fraction (1545), this corresponds to 1.6 CEMP stars, compared to the 33 in PIGS. Between $-3.0<[\mathrm{Fe} / \mathrm{H}]<-2.5$ we find a $[\mathrm{C} / \mathrm{Fe}]$ mean of 0.17 with a sigma of $0.25 \mathrm{dex}$. From such a Gaussian distribution, one would expect 2.6 CEMP stars out of 158 in this metallicity range, compared to 15 in PIGS. We therefore conclude that most CEMP stars in PIGS are real outliers of the $[\mathrm{C} / \mathrm{Fe}]$ distribution.

We want to stress here that we caution against interpreting the PIGS CEMP distribution and fractions at face value. In the next section we will discuss several uncertainties and systematic biases which will have to be taken into account before a solid conclusion on the CEMP fraction of the bulge (and its comparison to the halo) can be considered definitive.

\section{DISCUSSION}

\subsection{Carbon bias and selection function}

The selection of metal-poor stars in PIGS could be biased against CEMP stars, since carbon-rich stars have large molecular features in their spectra which affect the flux in the $\mathrm{CaHK}$ narrow-band and in the employed broad-band filters. The effect of carbon on the Pristine $\mathrm{CaHK}$ photometry and the PIGS colour-colour selection (example in Figure 2) in particular, are investigated in detail in Arentsen et al. (in prep.). In that study, synthetic photometry is derived from synthetic spectra with different carbon abundances to test the behaviour of carbon-rich stars. However, a direct comparison between the synthetic photometry and our PIGS selection is complicated. There are important caveats regarding the synthetic photometry due the assumption of local thermodynamic equilibrium (LTE) in one dimension (1D) for the stellar spectra, and simplified assumptions about the chemical compositions of the stars. Additionally, it is difficult to reconstruct the PIGS selection function. This means that even if it was perfectly known how carbon-rich stars behaved in the colour-colour space, there is still some uncertainty to which stars would and would not have been selected for follow-up. Below, we will briefly discuss the results from the synthetic photometry analysis and describe several challenges regarding the reconstruction of the PIGS selection function.

\subsubsection{Carbon-rich photometry}

Since there are more absorption features of carbon in the blue part of the spectrum, carbon-rich stars appear redder than carbon-normal stars (this effect has been known for a long time, e.g. Bond \& Neff 1969). In our synthetic photometry analysis, we indeed find that a CEMP star moves to the right in our colour-colour diagram, and could even move out of our selection box in that direction. The differences are larger for stars with higher carbon abundances and/or cooler temperatures (especially strong effects are seen for $T_{\text {eff }}<5000 \mathrm{~K}$ and $[\mathrm{C} / \mathrm{Fe}]>+1.5$ ). We also find that CEMP-s stars are affected more than CEMP-no stars, due to different relative $\mathrm{CNO}$ abundances.

In the y-axis of the colour-colour selection diagram, the behaviour of carbon-rich stars is more complicated (and different for CEMP-no and CEMP-s stars) due to the combination of three or four different filters. However, it appears that we have been "lucky" in selecting the colour combination in such a way that carbon-rich stars typically do not move down very much in our PIGS selection diagram. The synthetic stars are not leaking out of our selection box in that direction, but this can depend on the assumptions made.

Finally, since we applied a fixed magnitude cut and carbon-rich stars are fainter in the blue, there can be a slight selection bias against the faintest CEMP stars. This is expected to be worse for the PS1 selection where the magnitude cut is based on the bluer $g$ magnitude (4000 $\AA-5500 \AA)$ compared to the Gaia selection where the $G$ 
magnitude was used (which covers a much wider wavelength range, from $3400 \AA-10000 \AA$ ).

\subsubsection{PIGS selection function}

There are multiple challenges in reconstructing the selection function of PIGS follow-up targets. First, we wish to emphasise again that the high extinction towards the inner Galaxy makes studying this region (and determining a selection function) extremely challenging. The population selected for follow-up in PIGS will vary from field to field depending on the extinction, with (for example) varying contamination by more metal-rich stars. The extinction correction is especially difficult for the wide Gaia filters, but the PS1 correction is also far from perfect. The extinction map used (Green et al. 2019, or earlier versions) has variable quality throughout the footprint, has limited resolution, is affected by three-dimensional effects that are not taken into account in our analysis, and adopts a single extinction law (whereas it may in fact be variable).

Other factors that determine which $~ 360$ stars per $2 \mathrm{dF}$ field are selected for follow-up are, for example, the underlying metallicity distribution and the stellar density, which vary from field to field and depend strongly on the Galactic latitude. Additionally, not all the best targets are actually assigned a fibre in the fibre allocation process, since fibres can only be placed a certain distance from each other. The allocation also depended on the availability of $\mathrm{CaHK}$ photometry in a field (which was not always homogeneous) and the inhomogeneity of the extinction.

Furthermore, the reconstruction of our selection function is hampered by various changes throughout the survey. The goal was to keep improving our yields of the most metal-poor stars in the inner Galaxy, which is why these changes were made, but they result in an inhomogeneous survey selection. For example, we switched from Gaia to PS1 due to extinction challenges. The selection with the two surveys used different magnitude ranges, which roughly correspond to each other (but not perfectly). The discrepancy is higher for higher extinctions, since $g$ is much more affected than $G$. Other changes were made throughout the survey in the colour limits of the selection, in cuts on the parallax, in the way the extinction was treated, in the zero-point calibration of the $\mathrm{CaHK}$ photometry, and in the removal of variable stars.

\subsubsection{Halo Pristine samples}

Other Pristine-selected samples also have low CEMP fractions among giant stars (Aguado et al. 2019 corrected for $\log g$ effects, Caffau et al. 2020, Lucchesi et al. in prep.). However, these samples were selected much more strictly than the PIGS stars: stars were observed one by one based on their low photometric metallicities and not with a box selection as in PIGS. The selection effects are expected to be larger in the halo samples (this will be presented in more detail in Arentsen et al. in prep.).

\subsubsection{Summary}

There are several complications in reconstructing the PIGS selection function. Combined with the uncertainties on the modelling of synthetic spectra for CEMP stars (and hence uncertainties on synthetic photometry), we conclude that it is not possible to make solid predictions about which stars should or should not have ended up in our final PIGS follow-up sample. It is likely that some of the coolest, most carbon-enhanced stars were excluded from our selection, but it is not at all clear that this can fully explain the low CEMP fraction in PIGS. The generous box-selection of PIGS still contains many of the carbon-rich stars.

\subsection{Comparison with the literature}

\subsubsection{Halo samples}

Throughout this work, we have used the Placco et al. (2014) CEMP fraction as a reference. It is based on a compilation of $\sim 500 \mathrm{VMP}$ stars studied with high-resolution spectroscopy, which was expected to be unbiased with respect to carbon. The sample includes stars of various evolutionary phases, whereas PIGS contains only giant stars. To test whether there is a difference in the fraction between giants and turn-off/main sequence stars, we re-derive the CEMP fraction from the Placco et al. (2014) sample for giants only (2/3rd of the sample). For stars with $T_{\text {eff }}<5500 \mathrm{~K}$ and $\log g<4.0$, the fractions for $[\mathrm{Fe} / \mathrm{H}]<-2.0,-2.5,-3.0$ go down by $5 \%, 5 \%, 7 \%$, respectively, to $28 \%, 29 \%, 41 \%$. This does not solve the tension between PIGS and the Placco et al. (2014) fraction.

What about lower resolution spectroscopy studies in the halo? There have been a number of papers in recent years studying large samples of halo metal-poor stars at low/intermediate resolution. These samples consist of largely giant stars, and the authors typically report overall CEMP fractions in agreement with the high-resolution Placco et al. (2014) fraction (e.g. Placco et al. 2018, 2019; Yoon et al. 2018; Limberg et al. 2021). However, they sometimes do have a lack of the most carbon-rich CEMP stars, and they have varying overall distributions of CEMP stars on the $[\mathrm{Fe} / \mathrm{H}]-\mathrm{A}(\mathrm{C})$ diagram. It is beyond the scope of this paper to investigate these differences in detail, but they may be important.

\subsubsection{High-resolution inner Galaxy EMBLA sample}

The metal-poor inner Galaxy SkyMapper/EMBLA survey reported only one extremely metal-poor CEMP-no star with $[\mathrm{C} / \mathrm{Fe}]>+1.0$ and zero CEMP-s stars among their 33 stars with measured carbon abundances (Howes et al. 2015, 2016). We identified five additional CEMP stars in their sample (see Section 4.1).

Including the new CEMP stars, we find a CEMP fraction of $44 \%$ (4/9) for $[\mathrm{Fe} / \mathrm{H}]<-3.0$, consistent with the halo CEMP fraction of $48 \%$ (Placco et al. 2014) and the PIGS fraction of $42 \%$. For $[\mathrm{Fe} / \mathrm{H}]<-2.5$, the CEMP fraction is $18 \%(4 / 22)$, consistent with the PIGS fraction of $16 \%$ and the halo CEMP-no fraction of $24 \%$, but lower than the overall CEMP halo fraction of $34 \%$. For $[\mathrm{Fe} / \mathrm{H}]<$ -1.95 , including the two almost-CEMP stars, the CEMP fraction is $20 \%(6 / 30)$, lower than the halo fraction of $33 \%$ but higher than the PIGS fraction of $6 \%$. Only considering CEMP-no stars, the fraction is $17 \%(5 / 29)$, consistent with the CEMP-no halo fraction of $20 \%$.

The EMBLA sample is on average relatively cool with almost all stars having $T_{\text {eff }}<5000 \mathrm{~K}$, which is where photometric selection effects are stronger. However, due to their similar "box"-selection strategy as in PIGS, stars with moderate carbon-enhancement likely would not have been missed. Their lack of very carbon-rich CEMP stars (mainly CEMP-s) is potentially due to selection effects - the highest $[\mathrm{C} / \mathrm{Fe}]$ in EMBLA is +1.23 , all other CEMP stars have $[\mathrm{C} / \mathrm{Fe}]<+1.0$. The CEMP-no fraction in EMBLA appears to be consistent with the CEMP-no fraction in the literature, but the CEMPno stars in the literature have on average higher carbon abundances compared to those in EMBLA. 


\subsection{Abundance variations and 3D/non-LTE}

The exact distribution of $\mathrm{A}(\mathrm{C})$ in the PIGS CEMP stars can be affected by assumptions in the spectroscopic analysis. In the models used in the FERRE analysis, oxygen follows the alpha abundances and therefore $[\mathrm{O} / \mathrm{Fe}]=+0.4$, while nitrogen follows the solar abundance pattern and therefore $[\mathrm{N} / \mathrm{Fe}]=0.0$. In observed CEMP stars, there is a range of relative $\mathrm{CNO}$ abundances. For most stars this does not have a significant impact on the spectra as the strongest carbon feature is the $\mathrm{CH}$ G-band, but for cool and very carbon-rich (e.g. $[\mathrm{C} / \mathrm{Fe}]>+2.0$ ) stars other features such as $\mathrm{CN}$ and $\mathrm{C}_{2}$ start to become larger as well, and these depend on the $\mathrm{CNO}$ assumptions. Inspecting the fits of high $\mathrm{A}(\mathrm{C})$ stars we noticed that the $\mathrm{C}_{2}$ features were often weaker in the models compared to the data, although the $\mathrm{CH}$ G-band was well fitted. This potentially affects the FERRE $[\mathrm{C} / \mathrm{Fe}]$ derivation for such stars.

The synthetic grid we used for the analysis follows the simple 1D/LTE assumptions, which are usually also adopted for the analysis of CEMP stars in the halo. Recently, Norris \& Yong (2019) have cast doubts on the reported CEMP(-no) fractions in the literature. They found that once estimates for 3D and non-LTE corrections on iron and the $\mathrm{CH}$ G-band are taken into account (which are stronger for more metal-poor stars), the CEMP star carbon abundances decrease by up to $\sim 0.5 \mathrm{dex}$, reducing the number of CEMP-no stars by $\sim 70 \%$. Similar results were found by Amarsi et al. (2019), who reported lower carbon abundances in a careful 3D/non-LTE analysis of atomic carbon for a number of metal-poor stars. Our estimated CEMP fraction can also be affected by these effects. However, since the CEMP fractions in the halo and in PIGS are both based on similar assumptions, they can likely be compared without problem. One difference is that PIGS consists exclusively of giants, whereas literature samples are a mixture of dwarfs, turn-off stars and giants. These can be expected to be affected differently by 3D/non-LTE effects. However, even when limiting the high-resolution halo sample to only giants, there is a large discrepancy in the CEMP fractions (see the previous section).

\subsection{Interpretation of the PIGS CEMP population}

How can we interpret the population of CEMP stars in PIGS? There are strong differences between the CEMP fractions in PIGS and those known from the literature in the Galactic halo, which potentially reflect truths about the underlying stellar population and/or be related to selection effects. We discuss some possible interpretations for the CEMP-s and the CEMP-no stars.

\subsubsection{CEMP-s stars}

CEMP-s stars are expected to have interacted with a binary companion in the past, where a former AGB star has transferred carbon-rich material to the star we today see as carbon-enhanced. In the halo, roughly $10-13 \%$ of the stars with $[\mathrm{Fe} / \mathrm{H}]<-2.0$ are CEMP-s stars (Placco et al. 2014; Yoon et al. 2018). In PIGS, the distinction between CEMP-s and CEMP-no is not trivial because we have no sprocess abundance measurements. A rough separation can, however, be achieved by selecting stars with high $\mathrm{A}(\mathrm{C})$ to be CEMP-s, and stars with low $\mathrm{A}(\mathrm{C})$ as CEMP-no, as in Section 4.1. We have seen that there is a strong discrepancy between the CEMP population in PIGS and the halo in the $\mathrm{A}(\mathrm{C})>8.0$ regime. The PIGS sample has almost none of these stars, whereas they are abundant in the halo. The fraction of CEMP stars for $[\mathrm{Fe} / \mathrm{H}]<-2.0$ and $<-2.5$ is also discrepant with that in the halo. For $[\mathrm{Fe} / \mathrm{H}]<-2.0$, the overall
CEMP fraction in PIGS is only $6 \% \pm 0.5 \%$ (see Figure 9 ). Half of the CEMP stars in this range have $\mathrm{A}(\mathrm{C})>7.1$ and hence are CEMP-s by this definition.

The discrepancy with the halo CEMP-s fraction and the lack of stars with very high $\mathrm{A}(\mathrm{C})$ is potentially partly due to photometric selection effects, which are strongest at higher metallicities and higher carbon abundances. However, it is not certain that the selection effects are strong enough to fully account for such a low fraction. Based on the bias analysis in Arentsen et al. (in prep.), only a small percentage of CEMP-s stars is expected to move significantly in the colour-colour diagram in such a way as to fall outside of our photometric selection box. We might also have expected a higher CEMP fraction among warmer stars in our sample due to selection effects, which we do not observe.

Assuming that the photometric selection cannot fully account for the missing CEMP-s stars, there appears to be a real lack of CEMP$\mathrm{s}$ stars in the inner Galaxy. According to the population synthesis modelling of Abate et al. (2015b), there are several parameters that could change the fraction of CEMP-s stars by up to a few per cent; for example variations in the initial mass function, variations in the initial mass ratio and the period distribution of binary stars and the age of the population. Naturally a different binary fraction of the stars would also result in a difference in the number of CEMP-s stars. Not much is known about the number and the properties of binary stars in the inner Galaxy, let alone at the lowest metallicities.

The binary fraction of stars is expected to be lower in very highdensity environments (mainly because of dynamical interactions) this has been observed in globular clusters, which appear to have lower binary fractions compared to the field (e.g. Milone et al. 2012; Lucatello et al. 2015). D'Orazi et al. (2010) studied the occurrence of barium-rich stars ( $\mathrm{Ba} / \mathrm{CH} / \mathrm{CEMP}$ stars) in globular clusters of various metallicities, and found a much lower fraction of barium-rich stars in clusters compared to the field. Their sample contains five clusters with $[\mathrm{Fe} / \mathrm{H}] \leq-2.0$, with $50-100$ stars per cluster, and they find only one barium-rich star among all these. There appears to be a low CEMP-s fraction in globular clusters indeed.

The bulge population nowadays is not as dense as a globular cluster, but recent observations suggest that the contribution of dissolved globular clusters to the halo is significantly larger in the inner few $\mathrm{kpc}$ of the Milky Way than further out (Schiavon et al. 2017; Horta et al. 2021). The latter authors estimate that the fraction of the mass originating from globular clusters is $\sim 27 \%$ within $1.5 \mathrm{kpc}$, six times higher than at $10 \mathrm{kpc}$. Simulations also show that the fraction of stars in the inner Galaxy originating from globular clusters may be relatively large (Hughes et al. 2020). If many of the $[\mathrm{Fe} / \mathrm{H}]<-2.0$ inner Galaxy stars originated in globular clusters, like their more metal-rich counterparts at $-2.0<[\mathrm{Fe} / \mathrm{H}]<-1.0$ in Horta et al. (2021), their binary fraction can be lower than that of outer halo stars. This results in a lower CEMP-s fraction.

Yoon et al. (2018) and Lee et al. (2019) found that the local inner halo (close to the Sun) has relatively more CEMP-s stars than CEMP-no stars compared to the outer halo, but this possibly only represents the fact that the local inner halo is more metal-rich than the outer halo. They do not compare the fraction of CEMP-s stars between the inner and outer halo in a smaller metallicity interval (e.g. $-2.5<[\mathrm{Fe} / \mathrm{H}]<-2.0)$.

\subsubsection{CEMP-no stars}

There appears to be agreement between the CEMP fraction in PIGS and the overall literature fraction for $[\mathrm{Fe} / \mathrm{H}]<-3.0$, although we note that this is based on a relatively small sample of $\sim 15$ stars 
with unknown CEMP classes. Most literature CEMP stars in this metallicity range are CEMP-no stars: Placco et al. (2014) report a CEMP-no fraction of $43 \%$ and a CEMP-s fraction of only $5 \%$. In PIGS, three of the five CEMP stars in this range are most likely to be CEMP-no stars (when including the star very close to the $\mathrm{A}(\mathrm{C})$ division), but the other two overlap more with the CEMP-s stars in the $[\mathrm{Fe} / \mathrm{H}]-\mathrm{A}(\mathrm{C})$ diagram. Excluding the stars with $\mathrm{A}(\mathrm{C})>7.5$, the CEMP-no fraction for EMP stars is $25_{-10}^{+14} \%$, inconsistent with the literature CEMP-no fraction.

For $[\mathrm{Fe} / \mathrm{H}]<-2.5$, the fraction of CEMP-no stars in the Galactic halo is $24 \%$ (Placco et al. 2014), and the total fraction of CEMP stars in PIGS is $21 \%$. However, roughly half of the PIGS CEMP stars in this range have $\mathrm{A}(\mathrm{C})>7.1$ and likely belong to the CEMP-s category. The fraction of CEMP-no stars in this metallicity range therefore appears to be lower in PIGS than expected from the literature for the halo. For $[\mathrm{Fe} / \mathrm{H}]<-2.0$ this is most certainly the case, where $20 \%$ of the stars are expected to be CEMP-no but the overall fraction in PIGS is only $6 \%$ (and some of these can be CEMP-s stars).

Stars with $[\mathrm{Fe} / \mathrm{H}]<-3.0$ are not expected to be significantly affected by carbon-biases in the photometry, except for the coolest $\left(T_{\text {eff }}<4750 \mathrm{~K}\right)$ and most carbon-rich $([\mathrm{C} / \mathrm{Fe}]>+2.0)$ stars $(\mathrm{Ar}-$ entsen et al in prep.). Stars of these temperatures are only a minority in the PIGS sample, and stars with such high carbon abundances are a minority among the literature CEMP-no stars. It can therefore be expected that the CEMP-no fraction in this metallicity range is hardly affected by a photometric bias. For stars with $[\mathrm{Fe} / \mathrm{H}]<-2.0$ and $<-2.5$, slightly warmer and less carbon-rich stars are also affected, but still most of the CEMP-no stars are expected to stay within our selection.

Our observations suggest that the CEMP-no fraction in the inner Galaxy is inconsistent with the halo, with the discrepancy becoming larger at higher metallicities. The EMBLA inner Galaxy CEMP-no fraction may appear consistent with the halo, but their CEMP-no stars all have relatively low $[\mathrm{C} / \mathrm{Fe}]$. Assuming possible selection biases are not the (sole) cause of the PIGS and/or EMBLA discrepancies, we search for a process that reduces the inner Galaxy CEMP-no fraction, primarily at higher metallicities. The stars that ended up in the bulge region possibly formed in larger building blocks (which are able to retain more of their gas) compared to stars ending up in the diffuse halo (likely born in smaller building blocks, which quickly lose most of their gas). Chemical evolution proceeded more quickly in dense regions, which can for example be seen in the chemical abundances of bulge stars (for a review, see e.g. Barbuy et al. 2018). The Cenhanced signature from the First Stars is potentially still visible in the EMP stars because those formed very early on, but wiped out at higher metallicities due to the rapid chemical enrichment and mixing of the ISM with the ejecta from many Population II supernovae. This reduces the CEMP-no fraction at higher metallicities in the inner Galaxy. Additionally, to our best knowledge there are currently no CEMP-no stars known in globular clusters. If there has been a larger contribution of globular cluster stars to the inner Galaxy compared to the halo, as discussed in the previous section, this also reduces the CEMP-no fraction.

\subsection{Future work}

This work represents a significant step forward in the study of the population of CEMP stars in the inner Galaxy. We conclude that deriving unbiased CEMP fractions based on photometrically selected samples is an extremely challenging task. There are several avenues to investigate the inner Galaxy CEMP population further.

First, it will be crucial to do high-resolution spectroscopic follow- up of our CEMP candidates to get their detailed abundance patterns which will reveal information about their origin. Stars that became carbon-enhanced due to mass-transfer from a binary companion are expected to have high s-process abundances (e.g. strontium and barium). CEMP-no stars have typical abundance patterns which can be related to the properties of the First Stars and their supernovae that enriched the medium out of which they formed. The high A(C) stars around $[\mathrm{Fe} / \mathrm{H}]=-3.0$ are of particular interest, as they lie in a region of parameter space that holds both CEMP-no and CEMP-s stars. Curiously, Arentsen et al. (2019) have shown that binarity among CEMP-no stars in this $\mathrm{A}(\mathrm{C})$ regime is higher than among the CEMPno stars with lower $\mathrm{A}(\mathrm{C})$ (although this still has to be confirmed with larger samples). They hypothesise that some of these stars were born with less carbon initially, but have obtained additional carbon as their binary companion transferred it to them. These CEMP-no stars do not show the typical mass-transfer s-process abundance enhancements, but it is questionable whether AGB stars at such low metallicities do produce s-process elements in the same way stars at higher metallicities do (see Arentsen et al. 2019, and references therein). Therefore not only should the abundance patterns of our stars be determined, monitoring the radial velocity adds valuable information as well.

Secondly, we have not taken into account the distances and/or orbits of the stars in our sample. We have implicitly assumed that all of them are inner Galaxy stars, loosely defined as stars in the inner $4-5 \mathrm{kpc}$ of the Milky Way. However, some stars are potentially simply passing through the inner regions on highly elliptical orbits with large apocentres and are thus not truly inner Galaxy stars. It is still unclear how many of the metal-poor stars currently in the inner Galaxy are halo interlopers, with estimates ranging from $25-75 \%$ (Howes et al. 2015; Kunder et al. 2020; Lucey et al. 2021). It is desirable to have orbital information of all the stars in PIGS, to be able to select a true inner Galaxy sample. We will investigate the orbital properties of PIGS stars in a future work. Unfortunately, the inner Galaxy is too far away to have good parallax information in the latest Gaia data release (EDR3, Gaia Collaboration et al. 2020), which makes distance determinations challenging. Better distances can be estimated if photometric information is included, but this is challenging towards the bulge due to the high, inhomogeneous extinction. Additionally, this will be especially difficult for CEMP stars since their colours are affected by carbon.

Additional work on the radial variation in the CEMP fraction in the inner Galaxy and the rest of the halo and the relative occurrence of globular cluster stars is necessary to shed more light on the CEMP star population and what it can teach us about the build-up of the Milky Way. We do not study the radial variation in this paper, due to complications with the selection function of PIGS and the photometric bias for carbon-rich stars combined with the high extinction and unknown distances.

Finally, the details of the photometric selection of metal-poor candidates should be further investigated. The synthetic analysis of Arentsen et al. (in prep.) to test biases against carbon-rich stars is useful, but has its limits. There are several observational avenues that can shed light on the situation. The main Pristine survey will perform a large follow-up campaign using WEAVE (the William Herschel Telescope Enhanced Area Velocity Explorer, a multi-object spectroscopic facility in the Northern hemisphere, Dalton et al. 2018), that will result in an incredibly rich spectroscopic sample of very metalpoor stars, including measured carbon and s-process abundances. WEAVE does not cover the inner Galaxy, but the Southern hemisphere 4-metre Multi-Object Spectroscopic Telescope (4MOST, de Jong et al. 2019) will. The 4MIDABLE-LR consortium survey will target metal-poor stars in the inner Galaxy (Chiappini et al. 2019), 
partly pre-selected from PIGS. This will significantly enlarge the number of very metal-poor inner Galaxy stars with spectroscopic follow-up, including estimates for carbon and s-process abundances useful for the study of CEMP stars. The WEAVE and 4MOST samples may still be biased with respect to carbon, but the homogeneous selection, sample size and data quality should allow for a good quantification of the effect. A further promising avenue for producing less biased samples of CEMP stars may be the use of a set of photometric filters with the sensitivity to measure carbon, for example using a narrow-band filter covering the G-band. A first encouraging exploration of this approach has been done by Whitten et al. (2019, 2021), for the J-PLUS and S-PLUS surveys (Cenarro et al. 2019; Mendes de Oliveira et al. 2019). The dispersed BP/RP spectra in future Gaia releases may also be very useful for such an investigation.

\section{SUMMARY AND CONCLUSIONS}

In this paper, we have studied the occurrence of CEMP stars in the inner Galaxy using the Pristine Inner Galaxy Survey (PIGS). Our main results can be summarised as follows:

- The final PIGS spectroscopic sample after three years of followup contains 6700 stars with $[\mathrm{Fe} / \mathrm{H}]<-1.5$, of which 1900 have $[\mathrm{Fe} / \mathrm{H}]<-2.0$. This is the largest sample of very metal-poor stars in the inner Galaxy to date, and is well-suited for the study of CEMP stars in the central regions of the Milky Way.

- The carbon abundance of RGB stars in PIGS decreases with decreasing $\log g$, which is also seen in halo stars. The magnitude of the carbon depletion is consistent with previous models by Placco et al. (2014). We used those models to derive carbon corrections for the entire PIGS sample.

- We discovered 96 new CEMP stars in PIGS, which is more than a ten-fold increase in the number of known CEMP stars in the inner Galaxy, since only seven CEMP stars were previously found in the inner Galaxy (Howes et al. 2015, 2016; Koch et al. 2016). Of the PIGS CEMP stars, 72 lie in the CEMP-s range of the $[\mathrm{Fe} / \mathrm{H}]-\mathrm{A}(\mathrm{C})$ diagram, and there are 24 that are likely CEMP-no stars.

- The fraction of CEMP stars with respect to carbon-normal stars is lower in PIGS than in Galactic halo literature samples, except for stars with $[\mathrm{Fe} / \mathrm{H}]<-3.0$. As a function of metallicity, the cumulative CEMP fractions in PIGS are $42_{-13}^{+14} \%([\mathrm{Fe} / \mathrm{H}]<-3.0)$, $16_{-3}^{+3} \%([\mathrm{Fe} / \mathrm{H}]<-2.5)$ and $5.7_{-0.5}^{+0.6}([\mathrm{Fe} / \mathrm{H}]<-2.0)$. The CEMP stars that are lacking compared to halo samples are mainly the more metal-rich and more carbon-rich stars.

- We concluded that there are many uncertainties in the selection function of PIGS and in estimates of photometric selection biases against carbon-rich stars, hindering a conclusive prediction regarding which CEMP stars would have ended up in our selection and which would not have. To our best understanding, however, the carbon bias appears to be insufficient to fully explain the low CEMP fraction in PIGS.

- We speculate that a low fraction of CEMP-no stars (especially at higher metallicities) is potentially related to very quick chemical evolution in the high-mass building blocks that made up the inner Galaxy. A low fraction of CEMP-s stars in the inner Galaxy may be related to a lower binary fraction than in the rest of the halo, possibly due to a larger contribution from former globular cluster stars.

- In the future, more light will be shed on the properties of CEMP stars in the inner Galaxy through high-resolution follow-up of our new CEMP stars, orbital analysis of PIGS stars, further tests of biases in the Pristine selection, and through large samples of metal-poor inner Galaxy stars observed with 4MOST.

The work presented in this paper is only the start of a promising avenue studying the build-up of the old, metal-poor inner Galaxy using carbon-enhanced metal-poor stars.

\section{ACKNOWLEDGEMENTS}

We thank the Australian Astronomical Observatory, which have made these observations possible. We acknowledge the traditional owners of the land on which the AAT stands, the Gamilaraay people, and pay our respects to elders past and present. Based on data obtained at Siding Spring Observatory (via programs S/2017B/01, A/2018A/01, OPTICON 2018B/029 and OPTICON 2019A/045, PI: A. Arentsen and A/2020A/11, PI: D. B. Zucker).

Based on observations obtained with MegaPrime/MegaCam, a joint project of CFHT and CEA/DAPNIA, at the Canada-FranceHawaii Telescope (CFHT) which is operated by the National Research Council (NRC) of Canada, the Institut National des Science de l'Univers of the Centre National de la Recherche Scientifique (CNRS) of France, and the University of Hawaii.

AA and NFM gratefully acknowledge funding from the European Research Council (ERC) under the European Unions Horizon 2020 research and innovation programme (grant agreement No. 834148). NFM, VH and GK gratefully acknowledge support from the French National Research Agency (ANR) funded project "Pristine" (ANR-18-CE31-0017). DA thanks the Leverhulme Trust for financial support. DBZ acknowledges the support of the Australian Research Council through Discovery Project grant DP180101791. The work of VMP is supported by NOIRLab, which is managed by the Association of Universities for Research in Astronomy (AURA) under a cooperative agreement with the National Science Foundation. JIGH acknowledges financial support from the Spanish Ministry of Science and Innovation (MICINN) project AYA2017-86389-P. Horizon 2020: This project has received funding from the European Union's Horizon 2020 research and innovation programme under grant agreement No 730890. This material reflects only the authors views and the Commission is not liable for any use that may be made of the information contained therein.

The authors thank the International Space Science Institute, Bern, Switzerland for providing financial support and meeting facilities to the international team "Pristine".

This work has made use of data from the European Space Agency (ESA) mission Gaia (https://www. cosmos.esa.int/ gaia), processed by the Gaia Data Processing and Analysis Consortium (DPAC, https://www . cosmos . esa.int/web/gaia/dpac/ consortium). Funding for the DPAC has been provided by national institutions, in particular the institutions participating in the Gaia Multilateral Agreement.

The Pan-STARRS1 Surveys (PS1) and the PS1 public science archive have been made possible through contributions by the Institute for Astronomy, the University of Hawaii, the Pan-STARRS Project Office, the Max-Planck Society and its participating institutes, the Max Planck Institute for Astronomy, Heidelberg and the Max Planck Institute for Extraterrestrial Physics, Garching, The Johns Hopkins University, Durham University, the University of Edinburgh, the Queen's University Belfast, the Harvard-Smithsonian Center for Astrophysics, the Las Cumbres Observatory Global Telescope Network Incorporated, the National Central University of Taiwan, the Space Telescope Science Institute, the National Aeronautics and Space Administration under Grant No. NNX08AR22G is- 
sued through the Planetary Science Division of the NASA Science Mission Directorate, the National Science Foundation Grant No. AST-1238877, the University of Maryland, Eotvos Lorand University (ELTE), the Los Alamos National Laboratory, and the Gordon and Betty Moore Foundation.

This research made extensive use of the MATPLOtLIB (Hunter 2007), PANDAS (McKinney 2010), ASTROPY (Astropy Collaboration et al. 2013; Price-Whelan et al. 2018) and Dustmaps (Green 2018) Python packages, and of TopCAT (Taylor 2005).

\section{DATA AVAILABILITY}

The data underlying this article will be shared on reasonable request to the corresponding author.

\section{REFERENCES}

Abate C., Pols O. R., Karakas A. I., Izzard R. G., 2015a, A\&A, 576, A118

Abate C., Pols O. R., Stancliffe R. J., Izzard R. G., Karakas A. I., Beers T. C., Lee Y. S., 2015b, A\&A, 581, A62

Aguado D. S., González Hernández J. I., Allende Prieto C., Rebolo R., 2017, A\&A, 605, A40

Aguado D. S., et al., 2019, MNRAS, 490, 2241

Allende Prieto C., Beers T. C., Wilhelm R., Newberg H. J., Rockosi C. M., Yanny B., Lee Y. S., 2006, ApJ, 636, 804

Alvarez R., Plez B., 1998, A\&A, 330, 1109

Amarsi A. M., Nissen P. E., Asplund M., Lind K., Barklem P. S., 2019, A\&A, 622, L4

Arentsen A., Starkenburg E., Shetrone M. D., Venn K. A., Depagne É., McConnachie A. W., 2019, A\&A, 621, A108

Arentsen A., et al., 2020a, MNRAS, 491, L11

Arentsen A., et al., 2020b, MNRAS, 496, 4964

Astropy Collaboration et al., 2013, A\&A, 558, A33

Barbuy B., Chiappini C., Gerhard O., 2018, ARA\&A, 56, 223

Beers T. C., Christlieb N., 2005, ARA\&A, 43, 531

Bessell M., Bloxham G., Schmidt B., Keller S., Tisserand P., Francis P., 2011, PASP, 123, 789

Bisterzo S., Gallino R., Straniero O., Cristallo S., Käppeler F., 2010, MNRAS, 404,1529

Bond H. E., Neff J. S., 1969, ApJ, 158, 1235

Bonifacio P., et al., 2015, A\&A, 579, A28

Bonifacio P., et al., 2019, MNRAS, 487, 3797

Bressan A., Marigo P., Girardi L., Salasnich B., Dal Cero C., Rubele S., Nanni A., 2012, MNRAS, 427, 127

Caffau E., et al., 2017, Astronomische Nachrichten, 338, 686

Caffau E., et al., 2020, MNRAS, 493, 4677

Carollo D., Freeman K., Beers T. C., Placco V. M., Tumlinson J., Martell S. L., 2014, ApJ, 788, 180

Casey A. R., Schlaufman K. C., 2015, ApJ, 809, 110

Cenarro A. J., et al., 2019, A\&A, 622, A176

Chambers K. C., et al., 2016, arXiv e-prints, p. arXiv: 1612.05560

Charbonnel C., 1994, A\&A, 282, 811

Charbonnel C., Lagarde N., 2010, A\&A, 522, A10

Chiappini C., Hirschi R., Meynet G., Ekström S., Maeder A., Matteucci F., 2006, A\&A, 449, L27

Chiappini C., et al., 2019, The Messenger, 175, 30

Chiti A., Frebel A., Jerjen H., Kim D., Norris J. E., 2020, ApJ, 891, 8

Choi J., Dotter A., Conroy C., Cantiello M., Paxton B., Johnson B. D., 2016, ApJ, 823, 102

D’Orazi V., Gratton R., Lucatello S., Carretta E., Bragaglia A., Marino A. F., 2010, ApJ, 719, L213

Da Costa G. S., et al., 2019, MNRAS, 489, 5900

Dalton G., et al., 2018, in Proc. SPIE. p. 107021B, doi:10.1117/12.2312031

Demarque P., Woo J.-H., Kim Y.-C., Yi S. K., 2004, ApJS, 155, 667

Denissenkov P. A., 2010, ApJ, 723, 563
Dotter A., 2016, ApJS, 222, 8

Frebel A., Norris J. E., 2015, ARA\&A, 53, 631

Frebel A., et al., 2006, ApJ, 652, 1585

Frebel A., Simon J. D., Kirby E. N., 2014, ApJ, 786, 74

Gaia Collaboration et al., 2018, A\&A, 616, A1

Gaia Collaboration Brown A. G. A., Vallenari A., Prusti T., de Bruijne J. H. J., Babusiaux C., Biermann M., 2020, arXiv e-prints, p. arXiv:2012.01533 García Pérez A. E., et al., 2013, ApJ, 767, L9

Gratton R. G., Sneden C., Carretta E., Bragaglia A., 2000, A\&A, 354, 169

Green G., 2018, The Journal of Open Source Software, 3, 695

Green G. M., Schlafly E., Zucker C., Speagle J. S., Finkbeiner D., 2019, ApJ, 887,93

Gustafsson B., Edvardsson B., Eriksson K., Jørgensen U. G., Nordlund Å., Plez B., 2008, A\&A, 486, 951

Hansen T. T., Andersen J., Nordström B., Beers T. C., Placco V. M., Yoon J., Buchhave L. A., 2016a, A\&A, 586, A160

Hansen T. T., Andersen J., Nordström B., Beers T. C., Placco V. M., Yoon J., Buchhave L. A., 2016b, A\&A, 588, A3

Horta D., et al., 2021, MNRAS, 500, 5462

Howes L. M., et al., 2014, MNRAS, 445, 4241

Howes L. M., et al., 2015, Nature, 527, 484

Howes L. M., et al., 2016, MNRAS, 460, 884

Hughes M. E., Pfeffer J. L., Martig M., Reina-Campos M., Bastian N., Crain R. A., Kruijssen J. M. D., 2020, MNRAS, 491, 4012

Hunter J. D., 2007, Computing In Science \& Engineering, 9, 90

Kielty C. L., et al., 2020, arXiv e-prints, p. arXiv:2012.03960

Koch A., McWilliam A., Preston G. W., Thompson I. B., 2016, A\&A, 587, A124

Koesterke L., Allende Prieto C., Lambert D. L., 2008, ApJ, 680, 764

Koleva M., Prugniel P., Bouchard A., Wu Y., 2009, A\&A, 501, 1269

Kroupa P., 2001, MNRAS, 322, 231

Kroupa P., 2002, Science, 295, 82

Kunder A., et al., 2020, AJ, 159, 270

Lai D. K., Lee Y. S., Bolte M., Lucatello S., Beers T. C., Johnson J. A., Sivarani T., Rockosi C. M., 2011, ApJ, 738, 51

Lamb M., et al., 2017, MNRAS, 465, 3536

Lee Y. S., Beers T. C., Kim Y. K., Placco V., Yoon J., Carollo D., Masseron T., Jung J., 2017, ApJ, 836, 91

Lee Y. S., Beers T. C., Kim Y. K., 2019, ApJ, 885, 102

Lewis I. J., et al., 2002, MNRAS, 333, 279

Limberg G., et al., 2021, arXiv e-prints, p. arXiv:2103.07621

Longeard N., et al., 2018, MNRAS, 480, 2609

Longeard N., et al., 2020a, arXiv e-prints, p. arXiv:2005.05976

Longeard N., et al., 2020b, MNRAS, 491, 356

Lucatello S., Tsangarides S., Beers T. C., Carretta E., Gratton R. G., Ryan S. G., 2005, ApJ, 625, 825

Lucatello S., Sollima A., Gratton R., Vesperini E., D'Orazi V., Carretta E., Bragaglia A., 2015, A\&A, 584, A52

Lucey M., et al., 2019, MNRAS, 488, 2283

Lucey M., et al., 2021, MNRAS, 501, 5981

McKinney W., 2010, in Stéfan van der Walt Jarrod Millman eds, Proceedings of the 9th Python in Science Conference. pp $56-61$, doi:10.25080/Majora-92bf1922-00a

Mendes de Oliveira C., et al., 2019, MNRAS, 489, 241

Mészáros S., et al., 2012, AJ, 144, 120

Meynet G., Ekström S., Maeder A., 2006, A\&A, 447, 623

Milone A. P., et al., 2012, A\&A, 540, A16

Ness M., et al., 2013, MNRAS, 430, 836

Nomoto K., Kobayashi C., Tominaga N., 2013, ARA\&A, 51, 457

Norris J. E., Yong D., 2019, ApJ, 879, 37

Norris J. E., Gilmore G., Wyse R. F. G., Yong D., Frebel A., 2010, ApJ, 722, L104

Norris J. E., et al., 2013, ApJ, 762, 28

Placco V. M., Frebel A., Beers T. C., Stancliffe R. J., 2014, ApJ, 797, 21

Placco V. M., et al., 2018, AJ, 155, 256

Placco V. M., et al., 2019, ApJ, 870, 122

Plez B., 2008, Physica Scripta Volume T, 133, 014003

Price-Whelan A. M., et al., 2018, AJ, 156, 123 
Reggiani H., Schlaufman K. C., Casey A. R., Ji A. P., 2020, AJ, 160, 173

Roederer I. U., Preston G. W., Thompson I. B., Shectman S. A., Sneden C., Burley G. S., Kelson D. D., 2014, AJ, 147, 136

Salvadori S., Skúladóttir Á., Tolstoy E., 2015, MNRAS, 454, 1320

Saunders W., et al., 2004, AAOmega: a scientific and optical overview. pp 389-400, doi:10.1117/12.550871

Schiavon R. P., et al., 2017, MNRAS, 465, 501

Schlafly E. F., et al., 2016, ApJ, 821, 78

Schlegel D. J., Finkbeiner D. P., Davis M., 1998, ApJ, 500, 525

Sharp R., et al., 2006, Performance of AAOmega: the AAT multi-purpose fiber-fed spectrograph. p. 62690G, doi:10.1117/12.671022

Shetrone M., et al., 2019, ApJ, 872, 137

Skúladóttir Á., Tolstoy E., Salvadori S., Hill V., Pettini M., Shetrone M. D., Starkenburg E., 2015, A\&A, 574, A129

Spite M., Caffau E., Bonifacio P., Spite F., Ludwig H. G., Plez B., Christlieb N., 2013, A\&A, 552, A107

Stancliffe R. J., Glebbeek E., Izzard R. G., Pols O. R., 2007, A\&A, 464, L57

Stancliffe R. J., Church R. P., Angelou G. C., Lattanzio J. C., 2009, MNRAS, 396, 2313

Starkenburg E., Shetrone M. D., McConnachie A. W., Venn K. A., 2014, MNRAS, 441, 1217

Starkenburg E., et al., 2017, MNRAS, 471, 2587

Starkenburg E., et al., 2018, MNRAS, 481, 3838

Taylor M. B., 2005, in Shopbell P., Britton M., Ebert R., eds, Astronomical Society of the Pacific Conference Series Vol. 347, Astronomical Data Analysis Software and Systems XIV. p. 29

Tominaga N., Iwamoto N., Nomoto K., 2014, ApJ, 785, 98

Umeda H., Nomoto K., 2003, Nature, 422, 871

Vandenberg D. A., Smith G. H., 1988, PASP, 100, 314

Venn K. A., et al., 2020, MNRAS, 492, 3241

Whitten D. D., et al., 2019, A\&A, 622, A182

Whitten D. D., et al., 2021, arXiv e-prints, p. arXiv:2104.00016

Wilson E., 1927, Journal of the American Statistical Association, 22, 209

Wolf C., et al., 2018, Publ. Astron. Soc. Australia, 35, e010

Yong D., et al., 2013, ApJ, 762, 26

Yoon J., et al., 2016, ApJ, 833, 20

Yoon J., et al., 2018, ApJ, 861, 146

Youakim K., et al., 2017, MNRAS, 472, 2963

Youakim K., et al., 2020, MNRAS, 492, 4986

de Jong R. S., et al., 2019, The Messenger, 175, 3

This paper has been typeset from a $\mathrm{T}_{\mathrm{E}} \mathrm{X} / \mathrm{LT} \mathrm{E} \mathrm{X}$ file prepared by the author. 